\title{
Quantum Stochastic Processes
}

\author{
E. B. DAviEs ${ }^{\star}$ \\ School of Mathematics, Institute for Advanced Study, Princeton
}

Received May 25, 1969

\begin{abstract}
In order to describe rigorously certain measurement procedures, where observations of the arrival of quanta at a counter are made throughout an interval of time, it is necessary to introduce the concept of a quantum stochastic process. While fully quantum mechanical in nature, these have a great deal of similarity with classical stochastic processes and can be characterized by and constructed from their infinitesimal generators. The infinitesimal generators are naturally obtained from certain "fields" which we prove must be of the boson or fermion type.
\end{abstract}

\section{$\S 1$. Introduction}

In the generally accepted mathematical accounts of quantum mechanics the evolution of quantum systems is divided into two basically different types. In the first, the system is not observed and evolves according to a one parameter (time) unitary group of automorphisms of the given Hilbert space. The fact that this is taken to be a unitary group rather than a representation of the positive real line by isometries reflects the belief that the mathematical description of the evolution is essentially unchanged by time inversion. The second kind of evolution occurs during the process of measurement, which is supposed to occur at an instant of time determined by the experimenter. If the state of the system immediately before the measurement is represented by the trace class operator $\varrho$ and the observable is described by a self-adjoint operator $A=\sum_{n=1}^{\infty} \lambda_{n} P_{n}$ with discrete spectrum, then the state immediately after the measurement is generally taken to be $\varrho^{\prime}=\sum_{n=1}^{\infty} P_{n} \varrho P_{n}$. The map $\varrho \rightarrow \varrho^{\prime}$ takes pure states to mixed states and time inversion is impossible because $\varrho \rightarrow \varrho^{\prime}$ is not one-one. This is generally explained by the fact that measurement is an essentially irreversible process.

In recent years a class of optical experiments has been performed in which neither of these types of evolution is appropriate but where something very much more complicated seems to be required. The first of these experiments, performed by Hanbury, Brown, and Twiss, and later developments, described in $[1,2]$, were of the following type. A beam

$\star$ This work was supported by a National Science Foundation grant GP-7952X. 
of photons is split and directed at a small number of counters which detect the arrival of individual photons at random times. It turns out that the number of photons arriving at any such counter within a given interval of time is a random variable which depends strongly, for example, on whether the light is coherent or incoherent. It is possible to observe and calculate in a fully quantum mechanical way such random variables as the waiting time for the arrival of the next photon after a given one (see [2]). Secondly it turns out that there is a definite correlation between the random variables giving the photon counts of two different counters and that the correlation depends upon the state describing the photon beam (see $[1,3]$ ).

It is fairly clear that although these measurements can be predicted by quantum theory they do not fall within the usual description of measurement theory, and the occurrence of waiting times and correlation functions suggests that the proper mathematical framework for their description is a quantum mechanical theory of stochastic processes. In this paper we lay the foundations for such a theory and find the most general form of a certain class of processes, which correspond in the classical theory of stochastic processes most closely to the Markov jump processes. As in the classical theory this turns out to involve the analysis of certain representations of the additive semigroup of the positive real line, in this case by positive linear endomorphisms of the trace class operators.

In the last section of the paper we give a rather more physical description of the results. We show how the theory is related to the measuring processes of quantum optics and how the correlation functions for a set of photon counters may be derived from a certain stochastic process. Since there are certain technical difficulties involved with this we actually only discuss completely the quantum stochastic process related to a set of counters sensitive to the strength of a fermion field, but it can be seen that the results for this case are very similar to those for the boson field. It is not claimed that any of the examples corresponds fully to a particular physical system. Much as Gaussian processes are an idealization of Brownian motion, so the process here are obtained by an idealization of the quantum mechanical measuring process, but we can hope they will be reasonably good models for certain experimental situations where classical probability theory is not applicable.

\section{§ 2. The Basic Formulation}

We refer the reader to [4-6] for explanations of any undefined terms in this paper, and in particular for definitions of state spaces, observables, instruments, and the composition of instruments. Except in this section 
we shall only be interested in the case where the state space $(V, \tau)$ consists of $V=\mathscr{T}_{s}(\mathscr{H})$, the ordered Banach space (under the trace norm) of selfadjoint trace class operators on a Hilbert space $\mathscr{H}$, and $\tau=\operatorname{tr}$, the normalised trace on $V$. However, for the time being we shall formulate the idea of a stochastic process in the general situation. At the end of the section we give an example to show that our definition contains the theory of Markov jump processes in classical probability as a special case.

Suppose we are given an apparatus which records events, each event being represented by a point $(x, t)$ in $X \times(0, \infty)$, where $t$ is the instant at which the event occurs and $x \in X$, where $X$ is a separable locally compact Hausdorff space representing the set of all possible values of some observable the apparatus is measuring. A sample point is defined as a sequence of events $\left\{\left(x_{i}, t_{i}\right): i=1,2, \ldots\right\}$ such that $0<t_{1}<t_{2}<\cdots$ and either the sequence terminates or $t_{n} \rightarrow \infty$ as $n \rightarrow \infty$. The sample space $X_{\infty}$ is defined as the set of all sample points and is a Borel subset of

$$
\bigcup_{m=0}^{m=\infty}\left\{\prod_{n=0}^{m} X \times(0, \infty)\right\}
$$

is an obvious way. For each time $t>0$ we define the sample space $X_{t}$ as the set of all finite sequences $\left\{\left(x_{i}, t_{i}\right): i=1,2, \ldots, n\right\}$ such that $0<t_{1}<\cdots<t_{n} \leqq t$. $X_{t}$ is also a Borel space in a natural way and if $0<s<t \leqq \infty$ there is a Borel map $\pi$ of $X_{t}$ onto $X_{s}$ in which each sequence $\left\{\left(x_{i}, t_{i}\right): i=1, s, \ldots\right\}$ is taken to the subsequence formed by dropping all the events which occur after time $s$. Given any $s, t>0$ there is a oneone Borel isomorphism $\lambda$ from $X_{s} \times X_{t}$ onto $X_{s+t}$ defined by

$$
\lambda\left\{\left(x_{i}, s_{i}\right)_{i=1}^{m},\left(y_{j}, t_{j}\right)_{j=1}^{n}\right\}=\left(y_{1}, t_{1}\right) \ldots\left(y_{n}, t_{n}\right),\left(x_{1}, t+s_{1}\right) \ldots\left(x_{m}, t+s_{m}\right) .
$$

We now define a stochastic process on $X, V$ to be a family of instruments $\mathscr{E}$ defined on $X_{t}, V$ for all $t \geqq 0$ satisfying the following conditions

(i) $\mathscr{E}^{0}\left(X_{0}, \varrho\right)=\varrho$ for all $\varrho \in V$, where $X_{0}$ consists of the single sample point corresponding to a sequence of zero length,

(ii) for each $\varrho \in V, t \rightarrow \mathscr{E} t\left(X_{t}, \varrho\right)$ is continuous for all $t \geqq 0$.

(iii) for all $\varrho \in V$ and $s, t>0$

$$
\mathscr{E}^{\mathscr{t}}\left(F, \mathscr{E}^{s}(E, \varrho)\right) \equiv \mathscr{E}^{\mathscr{t}} \circ \mathscr{E}^{\mathscr{s}}(F \times E, \varrho)=\mathscr{E}^{s+t}(\lambda(F \times E), \varrho) .
$$

The last condition says that the evolution after time $t$ depends only on the state at time $t$, and the evolution is homogeneous in time. It is a generalization of the Chapman-Kolmogorov equation. It should be explained here that the instrument $\mathscr{E}$ is to be thought of as accepting a state $\varrho \in V$ at time zero and emitting an output state $\mathscr{E}(E, \varrho)$ conditional upon the set $E \leqq X_{t}$ at the later time $t$. 
Given a stochastic process we obtain a one-parameter semigroup of endomorphisms of $V$ as follows. If we define $T_{t}: V \rightarrow V$ by

$$
T_{t}(\varrho)=\mathscr{E}\left(X_{t}, \varrho\right)
$$

then $T_{t}$ is a bounded positive linear operator of $V$ into $V$ satisfying $\tau\left[T_{t}(\varrho)\right]=\tau[\varrho]$ for all $t \geqq 0$ and $\varrho \in V$. Since $\lambda\left(X_{s} \times X_{t}\right)=X_{s+t}$ we obtain $T_{s} T_{t}=T_{s+t}$ from the (generalized) Chapman-Kolmogorov equations. The continuity condition (ii) shows that $T_{t}$ is a strongly continuous oneparameter semigroup on $V$.

There is a second semigroup of endomorphisms of $V$ we can define. Writing $z$ for the sample point in $X_{t}$ which consists of zero events, we define the bounded positive operator $S_{t}: V \rightarrow V$ by

$$
S_{t}(\varrho)=\mathscr{E}^{t}(z, \varrho)
$$

It is easily seen that for all $t \geqq 0$ and $\varrho \in V^{+}$

$$
\tau\left[S_{t}(\varrho)\right] \leqq \tau[\varrho] .
$$

Moreover as $\lambda(z, z)=z$ we have $S_{s} S_{t}=S_{s+t}$ for all $s, t \geqq 0$.

It is necessary now to make a further assumption about the stochastic process, that there exists a constant $K<\infty$ such that for all $\varrho \in V^{+}$ and $t>0$

$$
\tau\left[\mathscr{E}\left(X_{t}-z, \varrho\right)\right] \leqq K t \tau[\varrho]
$$

which is interpreted by saying that the stochastic process has a bounded interaction rate. In order to proceed further we introduce the Borel sets

$$
\begin{aligned}
& A_{t}^{n}=\left\{\text { all sample points in } X_{t} \text { containing exactly } n \text { events }\right\} \\
& \left.B_{t}^{n}=\bigcup_{m=n}^{\infty} A_{t}^{n}=\text { all sample points in } X_{t} \text { containing at least } n \text { events }\right\}
\end{aligned}
$$

observing that

$$
A_{s+t}^{l}=\bigcup_{m+n=l} \lambda\left(A_{s}^{m} \times A_{t}^{n}\right) .
$$

Let $m \geqq n$ and let $F_{m}^{n}$ be the family of all subsets $a$ of $(1, \ldots, m)$ containing exactly $n$ points. For $a \in F_{m}^{n}$ and $1 \leqq r \leqq m$ define $C_{a, r} \leqq X_{m^{-1} t}$ by

Then define $D_{t, m}^{n} \cong X_{t}$ by

$$
C_{a, r}=\left\{\begin{array}{lll}
X_{m^{-1} t} & \text { if } & r \notin a \\
B_{m^{-1} t}^{1} & \text { if } & r \in a .
\end{array}\right.
$$

$$
D_{t, m}^{n}=\bigcup_{a \in F_{m}^{n}} \lambda\left(C_{a, 1} \times \cdots \times C_{a, m}\right) .
$$


Clearly $D_{t, m}^{n} \subseteq B_{t}^{n}$ but conversely the characteristic functions of these sets satisfy

$$
\chi\left(B_{t}^{n}\right)=\lim _{m \rightarrow \infty} \chi\left(D_{t, m}^{n}\right) .
$$

Now for any state $\varrho \in V^{+}$the hypothesis of a bounded interaction rate $K$ implies

$$
\tau\left[\mathscr{E}^{\mathscr{t}}\left(\chi\left(D_{t, m}^{n}\right), \varrho\right)\right] \leqq \frac{m ! K^{n} t^{n} \tau[\varrho]}{(m-n) ! n ! m^{n}} .
$$

Using the Fatou-Lebesgue theorem and fundamental properties of instruments we now obtain

$$
\tau\left[\mathscr{E}\left(B_{t}^{n}, \varrho\right)\right] \leqq(n !)^{-1} K^{n} t^{n} \tau[\varrho] .
$$

As $z=\lambda(z \times \cdots \times z)$ we have for all $\varrho \in V^{+}$

$$
\tau\left[\mathscr{E}^{t}(z, \varrho)\right] \geqq\left(1-\frac{K t}{m}\right)^{m} \tau[\varrho]
$$

and going to the limit as $m \rightarrow \infty$ gives

$$
\tau\left[S_{t}(\varrho)\right] \geqq e^{-K t} \tau[\varrho] .
$$

As the semigroup $T_{t}$ is strongly continuous and

$$
T_{t}(\varrho)=S_{t}(\varrho)+\mathscr{E}\left(B_{t}^{1}, \varrho\right)
$$

it follows from Eq. (2.5) that $S_{t}$ is also a strongly continuous one parameter semigroup on $V$. Using the projection mapping $\pi$ from $A_{t}^{1}=X \times(0, t]$ onto $X$ we define

by the equation

$$
\mathscr{J}^{t}: \mathscr{B}(X) \times V \rightarrow V
$$

$$
\mathscr{J}^{t}(E, \varrho)=t^{-1} \mathscr{E}^{t}\left(\pi^{-1}(E), \varrho\right)
$$

and observe that $\mathscr{J}^{t}$ satisfies all of the axioms for an instrument in [4] except that instead of

$$
\tau\left[\mathscr{J}^{t}(X, \varrho)\right]=\tau[\varrho]
$$

for all $\varrho \in V$ we have

$$
\tau\left[\mathscr{J}^{t}(X, \varrho)\right] \leqq K \tau[\varrho] \text { for all } \varrho \in V^{+} .
$$

We are now able to rewrite Eq. (6) as

$$
t^{-1}\left(T_{t} \varrho-\varrho\right)=t^{-1}\left(S_{t} \varrho-\varrho\right)+\mathscr{J}^{t}(X, \varrho)+o(t) .
$$

If we assume for the moment that $\mathscr{J}^{t}$ converges to a limit as $t \rightarrow 0$ and that the infinitesimal generators $\partial / \partial t$ of $T_{t}$ and $\mathrm{H}$ of $S_{t}$ have a common 
dense domain $\mathscr{D}$ then from (2.10) we obtain the following evolution equation

$$
\frac{\partial}{\partial t}(\varrho)=H(\varrho)+\mathscr{J}(X, \varrho)
$$

This equation is precisely the version of the Kolmogorov forward differential equation relevant to this generalized stochastic process.

In order to show concretely how our theory is related to classical stochastic processes, we indicate how it contains the theory of Markov processes of the pseudo-Poisson type with linear increments as a special case. I should like to thank J. T. Lewis for suggesting this example. Let $X$ be the real line $\mathbb{R}$ and let $V$ be the ordered Banach space of all bounded signed Borel measures on $X$. If we define $\tau$ on $V$ by $\tau(\mu)=\mu(X)$ then $\tau$ is a strictly positive linear functional on $V$ and $(V, \tau)$ is a state space, [4]. The set $\left\{\mu \in V^{+}: \tau(\mu)=1\right\}$ of normalised states in $V$ is precisely the set of probability measures on $\mathbb{R}$, so we are describing the Kolmogorov model of probability. Following the notation of $[7$, p. 311-320] suppose that we have a Markov jump process with linear increments. Suppose that between jumps $X(t)$ varies linearly at a rate $c$ and that the interaction rate is given by a constant $\alpha>0$; finally suppose the jumps when they occur are described by a stochastic kernel $K$. From $K, \alpha, c$ it is possible to construct a classical stochastic process, but we show how to construct a stochastic process in our sense of the term; the two constructions can be shown to be essentially equivalent.

First we define $S_{t}: V \rightarrow V$ by

$$
\left(S_{t} \mu\right)(E)=e^{-\alpha t} \mu(E-c t)
$$

and verify that $S$ is a strongly continuous semigroup on $V$ taking pure states to pure states. We define $\mathscr{J}$ on $X, V$ by

$$
\{\mathscr{J}(F, \mu)\}(E)=\alpha \int_{F} K(x, E) \mu(d x)
$$

and verify that $\mathscr{J}$ is a bounded stochastic kernel (in the sense of $\S 4$ ) satisfying

$$
\tau[\mathscr{J}(X, \mu)]=\{\mathscr{J}(X, \mu)\}(X)=\alpha \int_{X} K(x, X) \mu(d x)=\alpha \tau[\mu]
$$

for all $\mu \in V$. It is now possible to construct a stochastic process $\mathscr{E}$ with $S_{t}, \mathscr{J}$ as infinitesimal generators by similar methods to those of Theorem 4.7 since for all $\mu \in V$

$$
\frac{d}{d t} \tau\left[S_{t}(\mu)\right]=\frac{d}{d t} e^{-\alpha t} \tau[\mu]=-\alpha e^{-\alpha t} \tau[\mu]=-\tau\left[\mathscr{J}\left(X, S_{t}(\mu)\right)\right] .
$$


If $x \in X$ we let $\varepsilon_{x}$ be the probability measure concentrated at $x$ and define

$$
Q_{t}(x, \Gamma)=\left\{T_{t}\left(\varepsilon_{x}\right)\right\}(\Gamma)
$$

for any Borel set $\Gamma \leqq X$ and any $t \geqq 0$. In the special case $c=0$ Eq. (10) then becomes

$$
\frac{\partial}{\partial t} Q_{t}(x, \Gamma)=-\alpha Q_{t}(x, \Gamma)+\alpha \int_{X} Q_{t}(x, d z) K(z, \Gamma)
$$

which is precisely the Kolmogorov forward equation [7, p. 314].

Now that we have formulated the theory in the general setting we want to start a much more detailed analysis in the case of interest to quantum mechanics, where $V=\mathscr{T}_{s}(\mathscr{H})$ as described earlier. The main problem is to find reasonable conditions on a stochastic process which enable us to rigorously derive Eq. (2.11), and then to show how to reconstruct the process from this differential equation.

\section{§ 3. One-Parameter Semigroups}

In this section we derive some technical results we shall need about strongly continuous one-parameter semigroups of positive endomorphisms of $V=\mathscr{T}_{s}(\mathscr{H})$. The theorems and proofs are basically modelled on corresponding results for one-parameter groups as described in [8], but seem to us sufficiently different to require explicit presentation. Throughout this section $\mathscr{H}$ will denote a separable Hilbert space, $\mathscr{T}_{s}(\mathscr{H})$ will denote the ordered Banach space of self-adjoint trace class operators on $\mathscr{H}$ under the trace norm and tr will denote the normalised trace on $\mathscr{T}_{s}(\mathscr{H})$. We recall that the pure states of the positive cone $\mathscr{T}_{s}(\mathscr{H})^{+}$ of $\mathscr{T}_{s}(\mathscr{H})$ are the elements of the form $\xi \otimes \bar{\xi}$ where $\xi \in \mathscr{H}$. We also note that there is a one-one correspondence between the real linear transformations $S: \mathscr{T}_{s}(\mathscr{H}) \rightarrow \mathscr{T}_{s}(\mathscr{H})$ and the complex linear self-adjoint transformations $S: \mathscr{T}(\mathscr{H}) \rightarrow \mathscr{T}(\mathscr{H})$, and we shall not distinguish between the two. I should like to thank E. Størmer for remarks which enabled me to very much shorten the proof of the following theorem.

Theorem 3.1. If $S: \mathscr{T}_{s}\left(\mathscr{H}_{1}\right) \rightarrow \mathscr{T}_{S}\left(\mathscr{H}_{2}\right)$ is a positive linear mapping which takes pure states to pure states then either

(i) there exists a bounded complex linear operator $B: \mathscr{H}_{1} \rightarrow \mathscr{H}_{2}$ such that for all $\varrho \in \mathscr{T}_{s}\left(\mathscr{H}_{1}\right), S(\varrho)=B \varrho B^{*}$; or

(ii) there exists a bounded antilinear operator $B: \mathscr{H}_{1} \rightarrow \mathscr{H}_{2}$ such that for all $\varrho \in \mathscr{T}_{s}\left(\mathscr{H}_{1}\right), S(\varrho)=B \varrho B^{*}$; or

(iii) there exists a bounded complex linear positive operator $B \in \mathscr{L}_{s}\left(\mathscr{H}_{1}\right)^{+}$ and a vector $\xi \in \mathscr{H}_{2}$ such that for all $\varrho \in \mathscr{T}_{s}\left(\mathscr{H}_{1}\right), S(\varrho)=\operatorname{tr}[B \varrho] \xi \otimes \bar{\xi}$. 
We make the preliminary observation that the positivity of $S$ implies it is bounded [9].

Lemma 3.2. If the theorem holds whenever $S$ satisfies the further condition

$$
\left\{\varrho \in \mathscr{T}_{s}\left(\mathscr{H}_{1}\right)^{+}: S(\varrho)=0\right\}=0
$$

then it is generally true.

Proof. $S$ has an adjoint $S^{*}: \mathscr{L}_{s}\left(\mathscr{H}_{2}\right) \rightarrow \mathscr{L}_{s}\left(\mathscr{H}_{1}\right)$ which is a bounded positive linear operator. If we define $A=S^{*}(I)$ and let $P$ be the orthogonal projection in $\mathscr{H}_{1}$ onto the closure of the range of $A$ it is easy to verify that

$$
\left\{\varrho \in \mathscr{T}_{s}\left(\mathscr{H}_{1}\right)^{+}: S(\varrho)=0\right\}=\left\{\varrho \in \mathscr{T}_{s}\left(\mathscr{H}_{1}\right)^{+}: P \varrho=\varrho P=0\right\} .
$$

Let $\xi=\alpha_{1} e_{1}+\alpha_{2} e_{2}$ where $P e_{1}=0, P e_{2}=e_{2},\left\|e_{1}\right\|=1$ and $\left\|e_{2}\right\|=1$. Then $S\left(e_{1} \otimes \bar{e}_{1}\right)=0$ and $S\left(e_{2} \otimes \bar{e}_{2}\right)=a \otimes \bar{a}$ for some non-zero $a \in \mathscr{H}_{2}$. For all complex constants $\beta_{1}, \beta_{2}$

$$
\begin{aligned}
0 & \left.\leqq S\left\{\beta_{1} e_{1}+\beta_{2} e_{2}\right) \otimes\left(\beta_{1} e_{1}+\beta_{2} e_{2}\right)^{-}\right\} \\
& \leqq S\left\{\left(\beta_{1} e_{1}+\beta_{2} e_{2}\right) \otimes\left(\beta_{1} e_{1}+\beta_{2} e_{2}\right)^{-}+\left(\beta_{1} e_{1}-\beta_{2} e_{2}\right) \otimes\left(\beta_{1} e_{1}-\beta_{2} e_{2}\right)^{-}\right\} \\
& =S\left\{2 \beta_{1} \bar{\beta}_{1} e_{1} \otimes \bar{e}_{1}+2 \beta_{2} \bar{\beta}_{2} e_{2} \otimes \bar{e}_{2}\right\} \\
& =2\left|\beta_{2}\right|^{2} a \otimes \bar{a} .
\end{aligned}
$$

Therefore since $a \otimes \bar{a}$ is a pure state,

$$
S\left\{\left(\beta_{1} e_{1}+\beta_{2} e_{2}\right) \otimes\left(\beta_{1} e_{1}+\beta_{2} e_{2}\right)^{-}\right\}=K\left(\beta_{1}, \beta_{2}\right) a \otimes \bar{a} .
$$

It is easy to verify that $K$ is a non-negative quadratic form on $L=\operatorname{lin}\left(e_{1}, e_{2}\right)$ so there is a positive self-adjoint operator $K: L \rightarrow L$ such that for all $\beta_{1}, \beta_{2} \in \mathbb{C}$

$$
S\left\{\left(\beta_{1} e_{1}+\beta_{2} e_{2}\right) \otimes\left(\beta_{1} e_{1}+\beta_{2} e_{2}\right)^{-}\right\}=\left\langle K\left(\beta_{1} e_{1}+\beta_{2} e_{2}\right), \beta_{1} e_{1}+\beta_{2} e_{2}\right\rangle a \otimes \bar{a} .
$$

Since $\left\langle K e_{1}, e_{1}\right\rangle=0$ it follows by spectral theory that

$$
K\left(\beta_{1} e_{1}+\beta_{2} e_{2}\right)=\beta_{2} e_{2} .
$$

In particular we see that

$$
S(\xi \otimes \bar{\xi})=\alpha_{2} \bar{\alpha}_{2} a \otimes \bar{a}=S\left\{(P \xi) \otimes(P \xi)^{-}\right\} .
$$

It now follows by linearity that for all $\varrho \in \mathscr{T}_{s}\left(\mathscr{H}_{1}\right), S(\varrho)=S(P \varrho P)$. From this formula it is clear that if the theorem holds for the restriction of $S$ to $\mathscr{T}_{s}\left(P \mathscr{H}_{m}\right)$ it holds for $S$ itself.

Proof of Theorem. By the lemma we need only consider the case where $A=S^{*}(I)$ has dense range. Let $\mathscr{K}_{n} \subseteq \mathscr{H}_{1}$ be the range of the spectral projection $P_{n}$ of $A$ corresponding to the set $\{\lambda \in \mathbb{R}: \lambda n \geqq 1\}$. Then define 
$\psi_{n}: \mathscr{T}_{s}\left(\mathscr{K}_{n}\right) \rightarrow \mathscr{T}_{s}\left(\mathscr{H}_{2}\right)$ by the equation

$$
\psi_{n}(\varrho)=\varphi\left(A^{-1 / 2} \varrho A^{-1 / 2}\right) .
$$

$\psi_{n}$ is a positive bounded linear map such that for all $\varrho \in \mathscr{T}_{s}\left(\mathscr{K}_{n}\right)$

$$
\operatorname{tr}\left[\psi_{n}(\varrho)\right]=\operatorname{tr}\left[\left(A^{-1 / 2} \varrho A^{-1 / 2}\right) \varphi^{*}(I)\right]=\operatorname{tr}[\varrho] .
$$

Applying $\left[10\right.$, Lemma 5.4] to the adjoint map $\psi_{n}^{*}: \mathscr{L}_{s}\left(\mathscr{H}_{2}\right) \rightarrow \mathscr{L}_{s}\left(\mathscr{K}_{n}\right)$ we see that either (i)', there exists a bounded complex linear operator $B_{n}: \mathscr{K}_{n} \rightarrow \mathscr{H}_{2}$ such that $B_{n}^{*} B_{n}=P_{n} A P$ and for all $\varrho \in \mathscr{T}_{s}\left(\mathscr{K}_{n}\right)$ we have $S(\varrho)=B_{n} \varrho B_{n}$, or (ii)', the same holds but $B_{n}$ is antilinear, or (iii)', there exists a vector $\xi \in \mathscr{H}_{2}$ such that for all $\varrho \in \mathscr{T}_{s}\left(\mathscr{K}_{n}\right)$ we have $S(\varrho)=\operatorname{tr}[A \varrho] \xi \otimes \bar{\xi}$.

It is now clear that either $S$ is of type (iii) or there exists $N$ such that for all $n \geqq N$ the restriction of $S$ to $\mathscr{T}_{s}\left(\mathscr{K}_{n}\right)$ is of type (i)' or (ii)'. In the latter event for any $m \geqq n \geqq N$ and $\xi \in \mathscr{K}_{n}$,

$$
\left(B_{m} \xi\right) \otimes\left(B_{m} \xi\right)^{--}=\left(B_{n} \xi\right) \otimes\left(B_{n} \xi\right)^{-}
$$

so $B_{m} \xi=\lambda B_{n} \xi$ for some $|\lambda|=1$. Simple arguments show that $\lambda$ is independent of $\xi \in \mathscr{K}_{n}$ and that $B_{m}, B_{n}$ are both linear or both antilinear. It is now possible to multiply the $B_{r}$ by appropriate constants of absolute value one so that for all $m \geqq n, B_{m}$ is an extension of $B_{n}$. As $\left\|B_{n}\right\|^{2}=\left\|B_{n}^{*} B_{n}\right\|$ $=\left\|P_{n} A P_{n}\right\| \leqq\|A\|$, the common extension to $\bigcup_{n=1}^{\infty} \mathscr{K}_{n}$ is bounded and has a unique bounded extension to a bounded linear or antilinear operator $B$ on $\mathscr{H}_{1}$. Continuity arguments show that $S(\varrho)=B \varrho B^{*}$ for all $\varrho$ in $\mathscr{I}_{s}\left(\mathscr{H}_{1}\right)$.

The following lemma is a weakened version of the main theorem at the end of this section.

Lemma 3.3. Let $\mathscr{H}$ be a separable Hilbert space and let $S_{t}$ be a strongly continuous one-parameter semigroup of positive non-zero linear maps of $\mathscr{T}_{s}(\mathscr{H})$ into $\mathscr{T}_{s}(\mathscr{H})$ such that $S_{t}(\varrho)$ is a pure state for all $t \geqq 0$ whenever $\varrho$ is a pure state. Then there exists a family $A_{t}$ of bounded operators on $\mathscr{H}$ such that for all $\xi, \eta \in \mathscr{H}, t \rightarrow\left\langle A_{t} \xi, \eta\right\rangle$ is a Borel function on $[0, \infty)$, and a Borel multiplier $\gamma:[0, \infty) \times[0, \infty) \rightarrow\{z \in \mathbb{C}:|z|=1\}$ such that for all $s, t \geqq 0$

$$
A_{s+t}=\gamma(s, t) A_{s} A_{t}
$$

and for all $t \geqq 0$ and $\varrho \in \mathscr{T}_{s}(\mathscr{H})$

$$
S_{t}(\varrho)=A_{t} \varrho A_{t}^{*} .
$$

Proof. Except for the condition that all the above functions be Borel functions, this is a fairly easy consequence of Theorem 3.1. Let $\varrho \in \mathscr{T}_{s}(\mathscr{H})$ be a state which is not pure. By strong continuity of $S_{t}$ there is some $\delta>0$ such that for $0 \leqq t \leqq \delta, S_{t}(\varrho)$ is not pure, so for such $t, S_{t}$ is not of 
type (iii). Since $S_{2 t}=S_{t} \cdot S_{t}$ and the square of a mapping of type (ii) is of type (i), every $S_{t}$ for $0 \leqq t \leqq \delta$ is of type (i). The same now holds for all $t \geqq 0$ since we can always find an integer $n$ such that $0 \leqq t \leqq n \delta$. Now two non-zero mappings $\varrho \rightarrow B \varrho B^{*}$ and $\varrho \rightarrow A \varrho A^{*}$ are equal if and only if $B=\lambda A$ for some $|\lambda|=1$. Choosing some $A_{t}$ for each $t \geqq 0$ so that $S_{t}(\varrho)=A_{t} \varrho A_{t}^{*}$ we obtain $A_{s+t}=\gamma(s, t) A_{s} A_{t}$ for some $|\gamma(s, t)|=1$. Since we suppose $S_{t} \neq 0$ for all $t, \gamma(s, t)$ is unique and satisfies the multiplier equation

$$
\gamma(r, s) \gamma(r+s, t)=\gamma(r, s+t) \gamma(s, t)
$$

for all $r, s, t \geqq 0$.

To obtain the full result we make use of the theory of standard Borel spaces very much as in [8]. By estimates from semigroup theory $[11,12]$ on the rate of growth of $\left\|S_{t}\right\|$ there is no loss of generality in supposing that $\left\|S_{t}\right\| \leqq K<\infty$ for some constant $K$ and all $t \geqq 0$. Let $\Omega_{1} \leqq \mathscr{L}(\mathscr{H})$ be the set of all operators $A$ with $\|A\| \leqq K^{1 / 2}$. Under the strong operator topology $\Omega_{1}$ is a complete separable metrisable space. Let $\Omega_{2} \subseteq \mathscr{L}\left\{\mathscr{T}_{s}(\mathscr{H})\right\}$ be the set of all operators $A$ with $\|A\| \leqq K$. Under the strong operator topology $\Omega_{2}$ is also a complete separable metrisable space. Let $\pi: \Omega_{1} \rightarrow \Omega_{2}$ be the map defined by $(\pi A)(\varrho)=A \varrho A^{*}$. Then $\pi$ is continuous and the images of two non-zero elements $\omega, \omega^{\prime}$ of $\Omega_{1}$ are equal if and only if $\omega=\lambda \omega^{\prime}$ for some $|\lambda|=1$. It is immediate that the inverse image under $\pi$ of any point in $\Omega_{2}$ is closed and that the saturation $\pi^{-1} \pi(E)$ of any closed set $E \subseteq \Omega_{1}$ is Borel. From [13] there exists a Borel subset $F \cong \Omega_{1}$ such that $\lambda=\pi \mid F$ is a one-one Borel map of $F$ onto $\pi\left(\Omega_{2}\right)$. By [14] it follows that $\pi\left(\Omega_{1}\right)$ is a Borel set in $\Omega_{2}$ and that $\lambda$ is a Borel isomorphism of $F$ onto $\pi\left(\Omega_{1}\right)$. If now we define $A_{t}$ for all $t>0$ by $A_{t}=\lambda^{-1}\left(S_{t}\right)$ and $A_{0}=I$ it follows that for all $t \geqq 0$ and $\varrho \in \mathscr{T}_{s}(\mathscr{H}), S_{t}(\varrho)=A_{t} \varrho A_{t}^{*}$ and that for all $\xi, \eta \in \mathscr{H}, t \rightarrow\left\langle A_{t} \xi, \eta\right\rangle$ is a Borel function. If $\left\{e_{n}\right\}_{n=1}^{\infty}$ is an orthonormal basis for $\mathscr{H}$, as each $A_{t}$ is non-zero we can write $[0, \infty)$ as the disjoint union of a countable number of Borel sets $E_{m, n}$ such that for all $t \in E_{m, n}$, $\left\langle A_{t} e_{m}, e_{n}\right\rangle \neq 0$. Then

$$
\gamma \mid\left\{(s, t): s+t \in E_{m, n}\right\}=\left\langle A_{s} A_{t} e_{m}, e_{n}\right\rangle\left\langle A_{s+t} e_{m}, e_{n}\right\rangle^{-1}
$$

from which we see that $\gamma$ is a Borel function.

Theorem 3.4. If $\gamma$ is a Borel multiplier on $[0, \infty) \times[0, \infty)$ then there exists a Borel function $\lambda$ from $[0, \infty)$ to $\{z \in \mathbb{C}:|z|=1\}$ such that for

$$
\begin{gathered}
\text { all } s, t \geqq 0 \\
\gamma(s, t)=\lambda(s) \lambda(t) \lambda(s+t)^{-1} .
\end{gathered}
$$

Proof. Theorems of this kind for multipliers on locally compact topological groups are well known - see $[8,15,16]$ - but the proofs involve the construction of central group extensions, which do not seem easy 
to generalize to semigroups. We adopt the alternative method of proving that every Borel multiplier on $[0, \infty)$ can be extended to a Borel multiplier on $(-\infty, \infty)$. The existence of a suitable function $\lambda$ then follows from [15]. The method we use for extending the multiplier applies without alteration if we replace $[0, \infty)$ by the semigroup of positive elements of a totally ordered abelian group, but it would be interesting to obtain more general results.

For our purposes we can define a Borel multiplier on $[0, \infty)$ as a Borel function $\gamma$ from $[0, \infty) \times[0, \infty)$ to $\{z \in \mathbb{C}:|z|=1\}$ such that

$$
\begin{aligned}
\gamma(r, s) \gamma(r+s, t) & =\gamma(r, s+t) \gamma(s, t), \\
\gamma(s, 0) & =\gamma(0, s)=1
\end{aligned}
$$

for all $r, s, t \in[0, \infty)$. The definition of the extension $\bar{\gamma}$ on $(-\infty, \infty)$ $\times(-\infty, \infty)$ falls into six cases depending upon the signs of $a, b,(a+b)$. Specifically $\bar{\gamma}$ is defined by the following table.

\begin{tabular}{llll}
\hline $\operatorname{sign}(a)$ & $\operatorname{sign}(b)$ & $\operatorname{sign}(a+b)$ & $\bar{\gamma}(a, b)$ \\
\hline+ & + & + & $\gamma(a, b)$ \\
+ & - & + & $\gamma(a+b,-b)^{-1}$ \\
+ & - & - & $\gamma(-a-b, a)$ \\
- & + & + & $\gamma(-a, a+b)^{-1}$ \\
- & + & - & $\gamma(b,-a-b)$ \\
- & - & - & $\gamma(-b,-a)^{-1}$ \\
\hline
\end{tabular}

It is immediate from the definition that $\bar{\gamma}$ is a Borel function and that

$$
\bar{\gamma}(0, s)=\bar{\gamma}(s, 0)=1
$$

for all real $s$. The verification of the multiplier equation apparently requires the consideration of 64 cases but we can reduce these to a manageable number by use of the symmetries of the situation.

First observe that $\bar{\gamma}(x, z)=\bar{\gamma}(-z,-x)^{-1}$ for all real $x, z$. If we can find $a, b, c$ such that

$$
\bar{\gamma}(x, y) \bar{\gamma}(x+y, z)=\bar{\gamma}(x, y+z) \bar{\gamma}(y, z)
$$

is satisfied for $x=a, y=b, z=c$, it follows that (i) is also satisfied for $x=-c, y=-b, z=-a$. Therefore if (i) holds whenever $y \geqq 0$, it holds in all cases.

Now suppose we know that for all multipliers $\gamma$, Eq. (i) holds whenever $y \geqq 0$ and $x \geqq z$. Given any multiplier $\gamma$ we define the reversed multiplier $\lambda$ by $\lambda(x, y)=\gamma(y, x)$ and see that $\bar{\lambda}(x, y)=\bar{\gamma}(y, x)$ for all real $x, y$. If $a \leqq c$ and $b \geqq 0$ then Eq. (i) holds for the multiplier $\lambda$ and $x=c$, $y=b, z=a$, so Eq. (i) holds for the multiplier $\gamma$ and $x=a, y=b, z=c$. 
We now have only to verify Eq. (i) in the cases $y \geqq 0$ and $x \geqq z$. There are precisely eight cases, after eliminating logical impossibilities, depending on the signs of $x, y, z, x+y, y+z, x+y+z$. These are given from the following table.

\begin{tabular}{rllllll}
\hline & $\operatorname{sign}(x)$ & $\operatorname{sign}(y)$ & $\operatorname{sign}(z)$ & $\operatorname{sign}(x+y)$ & $\operatorname{sign}(y+z)$ & $\operatorname{sign}(x+y+z)$ \\
\hline (i) & + & + & + & + & + & + \\
(ii) & + & + & - & + & + & + \\
(iii) & + & + & - & + & - & + \\
(iv) & + & + & - & + & - & - \\
(v) & - & + & - & + & + & + \\
(vi) & - & + & - & + & + & - \\
(vii) & - & + & - & + & - & - \\
(viii) & - & + & - & - & - & - \\
\hline
\end{tabular}

In each of these cases Eq. (i) is verified by direct computation. In Case (vi) for example:

$$
\begin{aligned}
& \bar{\gamma}(x, y) \bar{\gamma}(x+y, z) \bar{\gamma}(x, y+z)^{-1} \bar{\gamma}(y, z)^{-1} \\
& =\gamma(-x, x+y)^{-1} \gamma(-x-y-z, x+y) \gamma(y+z,-x-y-z)^{-1} \gamma(y+z,-z) \\
& =\left\{\gamma(y+z,-x-y-z) \gamma(-x, x+y) \gamma(y+z,-z)^{-1} \gamma(-x-y-z, x+y)^{-1}\right\}^{-1} \\
& =\left\{\gamma(a, b) \gamma(a+b, c) \gamma(a, b+c)^{-1} \gamma(b, c)^{-1}\right\}^{-1}=1
\end{aligned}
$$

where $a=y+z \geqq 0, b=-x-y-z \geqq 0, c=x+y \geqq 0$.

Theorem 3.5. Let $\mathscr{H}$ be a separable Hilbert space and let $S_{t}$ be a strongly continuous one-parameter semigroup of positive non-zero linear maps of $\mathscr{T}_{s}(\mathscr{H})$ into $\mathscr{T}_{s}(\mathscr{H})$ such that $S_{t}(\varrho)$ is a pure state for all $t \geqq 0$ whenever $\varrho$ is a pure state. Then there exists a strongly continuous one-parameter semigroup of bounded operators $B_{t}$ on $\mathscr{H}$ such that for all $t \geqq 0$ and all $\varrho \in \mathscr{T}_{s}(\mathscr{H})$

$$
S_{t}(\varrho)=A_{t} \varrho A_{t}^{*}
$$

Proof. Given $\gamma, A_{t}$ as in Lemma 3.4 and $\lambda$ as in Theorem 3.4 we define $B_{s}=\lambda(s)^{-1} A_{s}$ and see that $B_{s} B_{t}=B_{s+t}$ for all $s, t \geqq 0$, while $s \rightarrow\left\langle B_{s} \xi, \eta\right\rangle=\lambda(s)^{-1}\left\langle A_{s} \xi, \eta\right\rangle$ is a Borel function on $[0, \infty)$ for all $\xi, \eta \in \mathscr{H}$. Now given any $\xi \in \mathscr{H}$

$$
\lim _{s \rightarrow 0}\left(B_{s} \xi\right) \otimes\left(B_{s} \xi\right)^{-}=\lim _{s \rightarrow 0} S_{s}(\xi \otimes \bar{\xi})=\xi \otimes \bar{\xi}
$$

is the trace norm by the strong continuity of $S_{s}$. Therefore $\bigcup_{t>0} B_{t}(\mathscr{H})$ is a dense subspace of $\mathscr{H}$. The strong continuity of the semigroup $B_{t}$ now follows from standard results on semigroups of operators $[11,12]$. 


\section{$\S$ 4. Structure of Stochastic Processes}

We now return to the analysis of stochastic processes begun in Section 2. Throughout this section we shall suppose $V=\mathscr{T}_{s}(\mathscr{H})$ where $\mathscr{H}$ is a given separable Hilbert space, and we shall suppose the stochastic process has an interaction rate bounded by the constant $K<\infty$. The one-parameter semigroup $T_{t}$ has the property that for any countable disjoint cover of $X_{t}$ by Borel sets $E_{n}, n=1,2, \ldots$ and any $\varrho \in V^{+}$

$$
T_{t}(\varrho)=\mathscr{E} t\left(X_{t}, \varrho\right)=\sum_{n=1}^{\infty} \mathscr{E}\left(E_{n}, \varrho\right) .
$$

It is apparent from this equation that we can expect, except in totally degenerate cases, that $T_{t}$ will transform pure states into mixed states and that as $t \rightarrow \infty, T_{t}(\varrho)$ will become more and more mixed. Such an argument does not apply to the semigroup $S_{t}$. Certainly as $t$ increases, $\varrho \rightarrow S_{t}(\varrho)$ is a transformation which is giving us more and more information about $\varrho$, but this information is of rather a minimal kind, that a certain type of interaction between the quantum system and the measuring apparatus has not occurred up to time $t$. It is therefore reasonable, though by no means necessary, to suppose that the evolution represented by $S_{t}$ is of the "simplest kind", which we shall interpret to mean in physical terms that if $\varrho$ is a pure state so is $S_{t}(\varrho)$ for all $t \geqq 0$. By the work of the previous section there exists a strongly continuous semigroup $B_{t}$ on $\mathscr{H}$ such that

Since

$$
S_{t}(\varrho)=B_{t} \varrho B_{t}^{*} .
$$

$$
e^{-K t} \operatorname{tr}[\varrho] \leqq \operatorname{tr}\left[S_{t}(\varrho)\right] \leqq \operatorname{tr}[\varrho]
$$

for all $\varrho \in V^{+}$we see that

$$
e^{-\frac{1}{2} K t}\|\xi\| \leqq\left\|B_{t} \xi\right\| \leqq\|\xi\|
$$

for all $\xi \in \mathscr{H}$ and $t \geqq 0$. We shall say more about the infinitesimal generator $Z$ of $B_{t}$ later.

The following lemma will be needed in the proof of Theorem 4.2.

Lemma 4.1. Let $B_{t}$ be a strongly continuous bounded semigroup of operators on $\mathscr{H}$ and let $S_{t}$ be the corresponding strongly continuous semigroup of operators on $V=\mathscr{T}_{s}(\mathscr{H})$. If $\varrho_{1} \in V^{+}$is such that

$$
t_{n}^{-1}\left(B_{t_{n}} \varrho_{1} B_{t_{n}}^{*}-\varrho_{1}\right) \equiv t_{n}^{-1}\left(S_{t_{n}}\left(\varrho_{1}\right)-\varrho_{1}\right)
$$

converges in the weak operator topology to a limit in $V$ for some sequence $t_{n} \rightarrow 0$, then $\varrho_{1}$ is in the domain $\mathscr{D}_{H}$ of the infinitesimal generator $H$ of $S_{t}$. 
Proof. We proceed by a slight variation of an argument in [12, p. 240]. If $\mathscr{D}^{\prime} \subseteq V$ is defined as the subspace

$$
\mathscr{D}^{\prime}=\left\{\varrho \in V: t_{n}^{-1}\left\{S_{t_{n}}(\varrho)-\varrho\right\}\right.
$$

converges in the weak operator topology to a limit in $V\}$

and $H^{\prime}(\varrho)$ is defined for $\varrho \in \mathscr{D}^{\prime}$ as the said limit, then $\mathscr{D}^{\prime} \supseteqq \mathscr{D}_{H}$ and $H^{\prime}$ is an extension of $H$. If we can show that $\left(I-H^{\prime}\right)$ is one-one then as $(I-H)$ maps $\mathscr{D}_{H}$ one-one onto $V$, it follows that $\mathscr{D}_{H}=\mathscr{D}^{\prime}$. Note that since each $S_{t}: V \rightarrow V$ is continuous for the weak operator topology, $\mathscr{D}^{\prime}$ is invariant under the action of $S_{t}$.

Suppose there exists some non-zero $\varrho \in V$ such that $\left(I-H^{\prime}\right) \varrho=0$, that is $H^{\prime} \varrho=\varrho$. Let $\varphi$ be a weakly continuous functional on $V$ such that $\varphi(\varrho)=1$, for example some constant multiple of a suitable vector state, and define $f:[0, \infty) \rightarrow(-\infty, \infty)$ by $f(t)=\varphi\left\{S_{t}(\varrho)\right\}$. Then $f$ is continuous, $f(0)=1$, and

$$
\begin{aligned}
\lim _{t_{n} \rightarrow 0} t_{n}^{-1} & \left\{f\left(t+t_{n}\right)-f(t)\right\} \\
& =\lim _{t_{n} \rightarrow 0} \varphi\left\{t_{n}^{-1}\left(S_{t+t_{n}}(\varrho)-S_{t}(\varrho)\right)\right\} \\
& =\lim _{t_{n} \rightarrow 0} \varphi\left\{S_{t}\left\{t_{n}^{-1}\left(S_{t_{n}}(\varrho)-\varrho\right)\right\}\right\} \\
& =\varphi\left\{S_{t}\left(H^{\prime} \varrho\right)\right\}=f(t) .
\end{aligned}
$$

The set $\left\{t \geqq 0: f(t) \geqq e^{\frac{1}{2} t}\right\}$ is non-empty, closed, and has no right endpoint. This implies it is unbounded which contradicts the fact that $f$ is a bounded function; this contradiction establishes the lemma.

Theorem 4.2. The domains of the infinitesimal generators of $S_{t}$ and $T_{t}$ are equal. Moreover for all $\varrho \in V, \mathscr{J}^{t}(X, \varrho)$ converges in norm as $t \rightarrow 0$.

Proof. The positive operators $A_{t}: V \rightarrow V$ defined by

$$
A_{t}(\varrho)=t^{-1} \mathscr{E} t\left(A_{t}^{1}, \varrho\right) \equiv \mathscr{J}^{t}(X, \varrho)
$$

are uniformly bounded, $\left\|A_{t}\right\| \leqq K$. If $\varrho \in V^{+}$then $\left\{A_{t}(\varrho)\right\}_{t \rightarrow 0}$ are uniformly bounded in the operator norm of $\mathscr{L}_{s}(\mathscr{H})$ and so there is a subsequence $t_{n} \rightarrow 0$ such that $A_{t_{n}}(\varrho)$ converges in the weak operator topology to a limit $\varrho \in \mathscr{L}_{s}(\mathscr{H})^{+}$. If $\left\{e_{n}\right\}_{n=1}^{\infty}$ is an orthonormal basis of $\mathscr{H}$ then

$$
\begin{aligned}
\operatorname{tr}\left[\varrho^{\prime}\right] & =\lim _{n \rightarrow \infty} \sum_{r=1}^{n}\left\langle\varrho^{\prime} e_{r}, e_{r}\right\rangle \\
& =\lim _{n \rightarrow \infty} \lim _{n \rightarrow \infty} \sum_{r=1}^{n}\left\langle A_{t_{n}}(\varrho) e_{r}, e_{r}\right\rangle \\
& \leqq K \operatorname{tr}[\varrho]<\infty
\end{aligned}
$$

so $\varrho^{\prime} \in V^{+}$. 
Now suppose $\varrho \in V^{\top} \cap \mathscr{D}_{H}$. By the above argument, Lemma 4.1, and Eq. (2.10) we see that $\varrho$ is in the domain of the infinitesimal generator of $T_{t}$. Again from Eq. (2.10) it follows that $\mathscr{J}^{t}(X, \varrho)$ converges in the norm topology as $t \rightarrow 0$. Now $V^{+} \cap \mathscr{D}_{I I}$ contains all finite sums $\sum_{r=1}^{r} \alpha_{r} \xi_{r} \otimes \bar{\xi}_{r}$ where $\alpha_{r} \geqq 0$ and $\xi_{r} \in \mathscr{D}_{Z}, Z$ being the infinitesimal generator of $B_{t}$, so $\left(V^{+} \cap \mathscr{D}_{H}-V^{+} \cap \mathscr{D}_{H}\right)$ is a dense linear subspace of $V$. Using the uniform boundedness of the operators $A_{t}$, it follows that $\mathscr{J}^{t}(X, \varrho)$ converges in norm as $t \rightarrow 0$ for all $\varrho \in V$. Again from Eq. (2.10) it follows that the domains of the infinitesimal generators of $S_{t}$ and $T_{t}$ are equal.

We next want to consider the convergence as $t \rightarrow 0$ of $\mathscr{J}^{t}(E, \varrho)$ for arbitrary $E \subseteq X$ and $\varrho \in V$. In order to use separability arguments, we are forced to change the problem somewhat. The $\mathscr{J}^{t}$ are not instruments in the sense of [4] because they do not satisfy the normalization conditions so we make the following slight modifications.

If $X$ is a separable locally compact Hausdorff space and $(V, \tau)$ is a state space, a bounded stochastic kernel $\mathscr{J}$ on $X, V$ can be defined in three possible ways

(S1) $\mathscr{J}_{1}$ is a bounded positive $\sigma$-additive measure on the $\sigma$-field of Borel sets in $X$ with values in $\mathscr{L}(V, V)$.

(S2) $\mathscr{J}_{2}$ is a bilinear map $\mathscr{J}_{2}: \mathscr{B}(X) \times V \rightarrow V$, where $\mathscr{B}(X)$ is the space of bounded Borel functions on $X$ such that

(i) if $f \in \mathscr{B}(X)^{+}$and $\varrho \in V^{+}$then $\mathscr{J}_{2}(f, \varrho) \in V^{+}$;

(ii) if $0 \leqq f_{n} \uparrow f$ in $\mathscr{B}(X)$ and $\varrho \in V^{+}$then $\mathscr{J}_{2}\left(f_{n}, \varrho\right)$ converges to $\mathscr{J}_{2}(f, \varrho)$ in norm.

(S3) $\mathscr{J}_{3}$ is a bilinear map $\mathscr{J}_{3}: K(X) \times V \rightarrow V$ where $K(X)$ is the space of continuous functions of compact support on $X$, such that

(i) if $f \in K(X)^{+}$and $\varrho \in V^{+}$then $\mathscr{J}_{3}(f, \varrho) \in V^{+}$;

(ii) for some constant $K<\infty$

$$
\left\|\mathscr{J}_{3}(f, \varrho)\right\| \leqq K\|\varrho\| \max \left\{\left|f^{\prime}(x)\right|: x \in X\right\} .
$$

These definitions are all equivalent in the same sense as in [6]. From now on we shall take the $\mathscr{J}^{t}$ as bounded stochastic kernels in the sense of (S3), the advantage being that $K(X)$ has a countable dense subset. The following lemma from operator theory will be needed in the proof of Lemma 4.4 .

Lemma 4.3. If $A_{n} \in \mathscr{T}_{s}(\mathscr{H})^{+}$is a sequence converging in the weak operator topology to $A \in \mathscr{T}_{s}(\mathscr{H})^{+}$then $A_{n}$ converges to $A$ in the trace norm if and only if $\operatorname{tr}\left[A_{n}\right]$ converges to $\operatorname{tr}[A]$.

Proof. The proof one way is trivial. Let us suppose that $A_{n} \rightarrow A$ in the weak operator topology and $\operatorname{tr}\left[A_{n}\right] \rightarrow \operatorname{tr}[A]$, and normalise to the case where $\operatorname{tr}\left[A_{n}\right] \leqq 1$ for all $n$. Given $0<\varepsilon<1$ let $P$ be a spectral projection 
of finite rank for $A$ such that $\|A-P A P\|<\varepsilon^{2}$. Since $P A_{n} P$ converges weakly to $P A P$ and $P$ is of finite rank, there exists $N$ such that $n \geqq N$ implies $\left\|P A_{n} P-P A P\right\|<\varepsilon^{2}$, and also $\left|\operatorname{tr}\left[A_{n}\right]-\operatorname{tr}[A]\right|<\varepsilon^{2}$. Then for such $n$

$$
\begin{aligned}
\left|\operatorname{tr}\left[A_{n}\right]-\operatorname{tr}\left[P A_{n} P\right]\right| \leqq & \left|\operatorname{tr}\left[A_{n}\right]-\operatorname{tr}[A]\right|+|\operatorname{tr}[A]-\operatorname{tr}[P A P]| \\
& +\left|\operatorname{tr}[P A P]-\operatorname{tr}\left[P A_{n} P\right]\right|<3 \varepsilon^{2} .
\end{aligned}
$$

If for some $n \geqq N, A_{n}$ has the spectral decomposition $\sum_{n=1}^{\infty} \lambda_{m} e_{m} \otimes \bar{e}_{m}$ where $\left\{e_{m}\right\}_{n=1}^{\infty}$ is an orthonormal basis for $\mathscr{H}$ then

$$
\begin{aligned}
\left\|A_{n}-P A_{n} P\right\| & \leqq\left\|A_{n}-P A_{n}\right\|+\left\|P A_{n}-P A_{n} P\right\| \\
& \leqq \sum_{m=1}^{\infty} \lambda_{m}\left\|\left(e_{m}-P e_{m}\right) \otimes \bar{e}_{m}\right\|+\sum_{m=1}^{\infty} \lambda_{m}\left\|P e_{m} \otimes\left(e-P e_{m}\right)^{-}\right\| \\
& \leqq 2 \sum_{m=1}^{\infty} \lambda_{m}\left\|e_{m}-P e_{m}\right\| \\
& \leqq 2\left\{\sum_{m=1}^{\infty} \lambda_{m}\right\}^{1 / 2}\left\{\sum_{m=1}^{\infty} \lambda_{m}\left\|e_{m}-P e_{m}\right\|^{2}\right\}^{1 / 2} \\
& =2\|A\|^{1 / 2}\left\{\sum_{m=1}^{\infty} \lambda_{m}\left(1-\left\|P e_{m}\right\|^{2}\right)\right\}^{1 / 2} \\
& \leqq 2\left\{\sum_{m=1}^{\infty} \lambda_{m}-\sum_{m=1}^{\infty} \lambda_{m}\left\|P e_{m}\right\|^{2}\right\}^{1 / 2} \\
& =2\left\{\operatorname{tr}\left[A_{n}\right]-\operatorname{tr}\left[P A_{n} P\right]\right\}^{1 / 2} \\
& <4 \varepsilon .
\end{aligned}
$$

Therefore for all $n \geqq N$

$$
\begin{aligned}
\left\|A-A_{n}\right\| & \leqq\|A-P A P\|+\left\|P A P-P A_{n} P\right\|+\left\|P A_{n} P-A_{n}\right\| \\
& <\varepsilon^{2}+\varepsilon^{2}+4 \varepsilon<6 \varepsilon,
\end{aligned}
$$

which proves the lemma.

Lemma 4.4. If $X$ is compact there exists a sequence $t_{n} \rightarrow 0$ of the form $t_{n}=2^{-m_{n}}$ and a bounded stochastic kernel $\mathscr{J}$ on $X$ such that for all $f \in K(X)$ and all $\varrho \in V, \mathscr{J}^{t_{n}}(f, \varrho)$ converges in norm to $\mathscr{J}(f, \varrho)$.

Proof. Using the separability of $K(X)$ and $V$, the uniform boundedness of $\mathscr{J}^{2-n}$, the compactness in the weak operator topology of $\{B \in \mathscr{L}(\mathscr{H})$ : $\|B\| \leqq 1\}$ and a diagonal selection argument we can find a sequence $t_{n}$ of the required form and a function $\mathscr{J}: K(X) \times V \rightarrow \mathscr{L}_{s}(\mathscr{H})$ such that for all $f \in K(X)$ and $\varrho \in V, \mathscr{J}^{t_{n}}(f, \varrho)$ converges to $\mathscr{J}(f, \varrho)$ in the weak operator topology. If $f \in K(X)^{+}$and $\varrho \in V^{+}$it follows that $\mathscr{J}(f, \varrho)$ 
$\in \mathscr{L}_{s}(\mathscr{H})^{+}$and

$$
\operatorname{tr}[\mathscr{J}(f, \varrho)] \leqq \liminf _{n \rightarrow \infty} \operatorname{tr}\left[\mathscr{J}^{t_{n}}(f, \varrho)\right] \leqq K\|f\|\|\varrho\|<\infty
$$

from which we see that $\mathscr{J}(f, \varrho) \in V$ and $\mathscr{J}$ is a bounded stochastic kernel. Also by Lemma 4.2 if $f \in K(X)^{+}$and $\varrho \in V^{+}$

$$
\begin{aligned}
\operatorname{tr}[\mathscr{J}(\|f\| 1, \varrho)] & =\lim _{n \rightarrow \infty} \operatorname{tr}\left[\mathscr{J}^{t_{n}}(\|f\| 1, \varrho)\right] \\
& \geqq \lim _{n \rightarrow \infty} \operatorname{tr}\left[\mathscr{J}^{t_{n}}(f, \varrho)\right]+\liminf _{n \rightarrow \infty} \operatorname{tr}\left[\mathscr{J}^{t_{n}}(\|f\| 1-f, \varrho)\right] \\
& \geqq \operatorname{tr}[\mathscr{J}(f, \varrho)]+\operatorname{tr}[\mathscr{J}(\|f\| 1-f, \varrho] \\
& =\operatorname{tr}[\mathscr{J}(\|f\| 1, \varrho)] .
\end{aligned}
$$

Therefore,

$$
\lim _{n \rightarrow \infty} \operatorname{tr}\left[\mathscr{J}^{t_{n}}(f, \varrho)\right]=\liminf _{n \rightarrow \infty} \operatorname{tr}\left[\mathscr{J}^{t_{n}}(f, \varrho)\right]=\operatorname{tr}[\mathscr{J}(f, \varrho)]
$$

and norm convergence follows from Lemma 4.3.

From now on we shall suppose that $X$ is compact, though we shall see in Theorem 4.6 that this is only a technical requirement. We define the total interaction rate $R \in \mathscr{L}_{s}(\mathscr{H})^{+}$as the unique operator such that for all $\varrho \in V$

$$
\operatorname{tr}[\varrho R]=\lim _{t \rightarrow \infty} \operatorname{tr}\left[\mathscr{J}^{t}(X, \varrho)\right]=\operatorname{tr}[\mathscr{J}(X, \varrho)] .
$$

Lemma 4.5. If $\xi \in \mathscr{D}_{Z}$ where $\mathscr{D}_{Z}$ is the domain in $\mathscr{H}$ of the infinitesimal generator $Z$ of the semigroup $B_{t}$ then

$$
\langle R \xi, \xi\rangle=-2 \operatorname{Re}\langle Z \xi, \xi\rangle \text {. }
$$

If $\varrho$ is an arbitrary state in $V$ then $\operatorname{tr}\left[S_{t}(\varrho)\right]$ is differentiable and

$$
\frac{d}{d t} \operatorname{tr}\left[S_{t}(\varrho)\right]=-\operatorname{tr}\left[R S_{t}(\varrho)\right] \text {. }
$$

Comments. This last equation gives a clear reason why we call $R$ the total interaction rate, since $\operatorname{tr}\left[S_{t}(\varrho)\right]$ is defined as the probability of no interaction between the quantum system and the measuring apparatus up to time $t$. We note that the interaction rate is independent of the state (supposing it is normalised) if and only if $R$ is a scalar multiple of the identity operator. This is clearly an unreasonable condition if the measuring apparatus is localised in some finite region of space.

Proof. If $\xi \in \mathscr{D}_{Z}$ then $\xi \otimes \bar{\xi} \in \mathscr{D}_{H}$ since

$$
\begin{aligned}
t^{-1}\left\{S_{t}(\xi \otimes \bar{\xi})-\xi \otimes \bar{\xi}\right\} & =t^{-1}\left\{\left(B_{t} \xi\right) \otimes\left(B_{t} \xi\right)^{-}-\xi \otimes \bar{\xi}\right\} \\
& =t^{-1}\left\{B_{t} \xi-\xi\right\} \otimes\left(B_{t} \xi\right)^{-}+\xi \otimes t^{-1}\left\{B_{t} \xi-\xi\right\}^{-} \\
& \rightarrow(Z \xi) \otimes \bar{\xi}+\xi \otimes(Z \xi)^{-}
\end{aligned}
$$


in norm as $t \rightarrow 0$. Taking the trace of both sides of Eq. (2.9) gives

$$
0=\operatorname{tr}\left[t^{-1}\left\{S_{t}(\xi \otimes \bar{\xi})-\xi \otimes \bar{\xi}\right\}\right]+\operatorname{tr}\left[\mathscr{J}^{t}(X, \xi \otimes \bar{\xi})\right]
$$

and going to the limit as $t \rightarrow 0$

$$
0=\langle Z \xi, \xi\rangle+\langle\xi, Z \xi\rangle+\operatorname{tr}[\mathscr{J}(X, \xi \otimes \bar{\xi})]
$$

which gives Eq. (4.2). Since $\xi \in \mathscr{D}_{Z}$ implies $B_{t} \xi \in \mathscr{D}_{Z}$ for all $t \geqq 0$, it follows that Eq. (4.3) holds for all

$$
\varrho \in L \equiv\left\{\sum_{r=1}^{n} \alpha_{r} \xi_{r} \otimes \bar{\xi}_{r}: \xi_{r} \in \mathscr{D}_{Z}, n=1,2, \ldots\right\} \cong V .
$$

Therefore for all such $\varrho \in L$

$$
\operatorname{tr}\left[S_{t}(\varrho)\right]=\operatorname{tr}[\varrho]-\int_{0}^{t} \operatorname{tr}\left[S_{s}(\varrho) R\right] d s .
$$

Since $L$ is dense in $V$ and Eq. (4.4) depends continuously on $\varrho$ it holds for all $\varrho \in V$ and differentiating this proves that Eq. (4.3) holds for all $\varrho \in V$.

We have now completed the preliminary work and can prove the main theorems of the section.

Theorem 4.6. Let $\mathscr{E}$ be a bounded stochastic process on $X, V$ where $X$ is a separable locally compact Hausdorff space and $V=\mathscr{T}_{s}(\mathscr{H})$ for a separable Hilbert space $\mathscr{H}$. If the semigroup $S_{t}$ takes pure states to pure states then $\mathscr{E}$ is uniquely determined by the infinitesimal generator $Z$ of the semigroup $B_{t}$ on $\mathscr{H}$ associated with $S_{t}$ and a bounded stochastic kernel $\mathscr{J}$ on $X$. Conversely $\mathscr{J}$ is uniquely determined by $\mathscr{E}, Z$ is uniquely determined by $\mathscr{E}$ except for addition of an arbitrary purely imaginary scalar multiple of the identity operator and $\mathscr{J}, Z$ are related by the equation

$$
\operatorname{tr}[\mathscr{J}(X, \xi \otimes \bar{\xi})]=-2 \operatorname{Re}\langle Z \xi, \xi\rangle
$$

where $\xi \in \mathscr{D}_{Z}$. We call $Z, \mathscr{J}$ the infinitesimal generators of the stochastic process.

Proof. If $\tilde{X}$ denotes the one-point compactification of $X$ then $X_{t}$ is a subset of $\tilde{X}_{t}$ and there is a natural extension of $\mathscr{E} t$ to a bounded stochastic process $\tilde{\mathscr{E}}$ on $\tilde{X}$ such that for all $\varrho \in V$ and $t \geqq 0$

$$
\tilde{\mathscr{E}}\left(\tilde{X}_{t}-X_{t}, \varrho\right)=0 \text {. }
$$

If $t>0, f \in K(\tilde{X})$ and $\varrho \in V$ then using the fact that $\tilde{\mathscr{E}} t$ has a bounded interaction rate we see that

$$
t \tilde{\mathscr{J}}^{t}(f, \varrho)=\lim _{n \rightarrow \infty} \sum_{r=1}^{\left[2^{m} t\right]} 2^{-m} S_{2-m\left\{\left[2^{m} t\right]-r\right\}} \tilde{\mathscr{J}}^{2-m}\left(f, S_{2-m(-1)} \varrho\right)
$$


where $\left[2^{m} t\right]$ denotes the largest integer not greater than $2^{m} t$. Using Lemma 4.4, the strong continuity of $S_{t}$ and the uniform boundedness of $S_{t}, t^{-1} \tilde{\mathscr{J}}^{t}$, it follows letting $m_{n} \rightarrow \infty$ that

$$
\tilde{\mathscr{J}}^{t}(f, \varrho)=t^{-1} \int_{0}^{t} S_{t-s} \tilde{\mathscr{J}}\left(f, S_{s} \varrho\right) d s
$$

for all $t \geqq 0, f \in K(\bar{X})$ and $\varrho \in V$, the integral existing as a vector-valued norm-convergent Ricmann integral. The above integral in fact exists in the same sense for all $f \in \mathscr{B}(\tilde{X})$ and using familiar dominated convergence arguments for the ordinary Lebesgue integral,

$$
\int_{0}^{t} \operatorname{tr}\left[S_{t-s} \tilde{\mathscr{J}}\left(f, S_{s} \varrho\right) B\right] d s
$$

where $B \in \mathscr{L}_{s}(\mathscr{H})$ is arbitrary, we see that Eq. (4.5) holds for all $f \in \mathscr{B}(\tilde{X})$. Therefore for all $\varrho \in V$ and $f \in \mathscr{B}(\tilde{X}), \tilde{\mathscr{J}}^{i}(f, \varrho)$ converges in norm to $\tilde{\mathscr{J}}(f, \varrho)$ as $t \rightarrow 0$. In particular $\tilde{\mathscr{J}}(\tilde{X}-X, \varrho)=0$. This greatly improves Lemma 4.4 , shows that it is not necessary to assume that $X$ is compact, and proves that $\mathscr{J}$ is uniquely determined by the stochastic process.

Now let $0<s_{1}<t_{1}<\cdots<s_{m}<t_{m} \leqq t$ and let $\left\{E_{i}\right\}_{i=1}^{m}$ be Borel subsets of $X$. Let $E \subseteq X_{t}$ be the Borel subset defined by

$$
\left.E=\left\{x_{i}, r_{i}\right)_{i=1}^{m}: s_{i}<r_{\iota} \leqq t_{i} \text { and } x_{i} \in E_{i}\right\}
$$

Then by its defining properties

$$
\begin{aligned}
\mathscr{E}(E, \varrho)= & \prod_{i=1}^{m}\left(t_{i}-S_{i}\right)\left\{S_{t-t_{m}} \mathscr{J}_{E_{m}}^{t_{m}-s_{m}} \ldots S_{s_{2}-t_{1}} \mathscr{J}_{E_{1}}^{t_{1}-s_{1}} S_{s_{1}} \varrho\right\} \\
= & \int_{s_{1}<r_{1} \leqq t_{i}} S_{t-t_{m}}\left(S_{t_{m}-r_{m}} \mathscr{J}_{E_{m}} S_{r_{m}-s_{m}}\right) \\
& \ldots S_{s_{2}-t_{1}}\left(S_{t_{1}-r_{1}} \mathscr{J}_{E_{1}} S_{r_{1}-s_{1}}\right) S_{s_{1}} \varrho d r_{1} \ldots d r_{m}
\end{aligned}
$$

or, simplifying

$\mathscr{E}(E, \varrho)=\int_{S_{t}<r_{i} \leqq t_{l}} S_{s-r_{m}} \mathscr{J}_{E_{m}} S_{r_{m}-r_{m-1}} \ldots S_{r_{2}-r_{1}} \mathscr{J}_{E_{1}} S_{r_{1}} \varrho d r_{1} \ldots d r_{m}$

This shows that $S_{t}, \mathscr{J}$ determine $\mathscr{E}$ because the sets $E$ of the given form constitute a semiring in the sense of [17] which generates the $\sigma$-field of all Borel subsets of $X_{t}$. The rest of the statements of the theorem are more or less immediate.

The following result shows that the stated conditions on $Z, \mathscr{J}$ are the only ones needed for them to be the infinitesimal generators of a (necessarily unique) stochastic process.

Theorem 4.7. Let $X$ be a separable locally compact Hausdorff space and $V=\mathscr{T}_{s}(\mathscr{H})$ for a separable Hilbert space. $\mathscr{H}$. Let $Z$ be the infinitesimal generator of a strongly continuous one parameter semigroup on $\mathscr{H}$ and 
let $\mathscr{J}$ be a bounded stochastic kernel on $X, V$ such that for all $\xi \in \mathscr{D}_{Z}$

$$
\operatorname{tr}[\mathscr{J}(X, \xi \otimes \bar{\xi})]=-2 \operatorname{Re}\langle Z \xi, \xi\rangle .
$$

Then there exists a unique stochastic process such that $Z, \mathscr{J}$ are its infinitesimal generators.

Proof. We define $\mathscr{E}^{\mathscr{t}}$ separately on each of the sets $A_{t}^{n} \cong X_{t}$. For the case $n=0$ we construct the semigroups $B_{t}, S_{t}$ from $Z$ and define $\mathscr{E} t(z, \varrho)$ $=S_{t}(\varrho)$. If $\varrho \in V^{+}$then repeating the calculations of Lemma 4.5 yields $\operatorname{tr}\left[S_{t}(\varrho)\right] \leqq \operatorname{tr}[\varrho]$. For $n \geqq 1$, using the correspondence $s_{i}=t_{i+1}-t_{i}$ where $t_{n+1}=t$ by convention, we can identify

$$
\begin{aligned}
A_{t}^{n}=\left\{\left(s_{n}, x_{n}, \ldots, s_{1}, x_{1}\right): 0<s_{1}, \ldots, s_{n-1}\right. \\
\text { and } \left.0 \leqq s_{n} \text { and } s_{1}+\cdots+s_{n}<t\right\} .
\end{aligned}
$$

We define the bounded stochastic kernel $\mathscr{G}$ on $(0, t], V$ by

$$
\mathscr{G}(E, \varrho)=\int_{0}^{t} \chi_{E}(s) S_{s}(\varrho) d s,
$$

noting that if $0 \leqq a<b \leqq t$ and $\varrho \in V^{+}$then

$$
\operatorname{tr}[\mathscr{G}\{(a, b], \varrho\}] \leqq(b-a) \operatorname{tr}[\varrho] .
$$

Following [4] we may define the composition $\mathscr{P}$ of $\mathscr{G}$ with $\mathscr{J}$ as a bounded stochastic kernel on $(0, t] \times X, V$. Now for any positive integers $m, r_{1}, \ldots, r_{n}$ we denote by $r$ the multiindex $\left(r_{1}, \ldots, r_{n}\right)$ and write $|r|=r_{1}+\cdots+r_{n}$. We define

$$
E_{m, r}=\left\{\left(s_{n}, x_{n}, \ldots, s_{1}, x_{1}\right): t\left(r_{i}-1\right)<2^{m} s_{i} \leqq t r_{i}\right\}
$$

so that for each $m$ as $r$ varies $\left\{E_{m, r}\right\}$ defines a partition of $\{(0, t] \times X\}^{n}$ and as $m$ increases the partition becomes finer and finer. We now define the bounded stochastic kernel $\mathscr{E}_{m}^{t}$ on $A_{t}^{n}, V$ by

$$
\mathscr{E}_{m}^{t}(E, \varrho)=\sum_{r} \mathscr{P}^{n}\left(E \cap E_{m, r}, S_{t-2-m_{t}|r|} \varrho\right)
$$

where $\mathscr{P}^{n}$ denotes the composition of $\mathscr{P}$ with itself $n$ times. If $m \geqq l$ then to each $r$ there exists a unique $k$ such that $E_{m, r} \leqq E_{l, k}$. For this $k$ we have

$$
2^{-l}\left(k_{i}-1\right) t \leqq 2^{-m}\left(r_{i}-1\right) t<2^{-m} r_{i} t \leqq 2^{-l} k_{i} t
$$

so that

and

$$
2^{-l}(|k|-n) \leqq 2^{-m}|r| \leqq 2^{-l}|k|
$$

$$
\left|\left(t-2^{-m} t|r|\right)-\left(t-2^{-l} t|k|\right)\right| \leqq 2^{-l} t n .
$$

Given $\varrho \in V$ and $\varepsilon>0$ we can now find $N=N(\varrho, \varepsilon)$ such that for all $m, r$ and $l, k$ satisfying $m \geqq l \geqq N$ and $E_{m, r} \leqq E_{l, k}$, if

$$
\sigma_{m, r} \equiv S_{t-2-m_{t}|r|}(\varrho)-S_{t-2-t_{t|k|}}(\varrho)
$$


then $\left\|\sigma_{m, r}\right\|<\varepsilon$. It follows that for all Borel sets $E \subseteq\{(0, t] \times X\}^{n}$

where

$$
\begin{aligned}
\left\|\mathscr{E}_{m} t(E, \varrho)-\mathscr{E}_{l}(E, \varrho)\right\| & =\left\|\sum_{r} \mathscr{P}^{n}\left(E \cap E_{m, r}, \sigma_{m, r}\right)\right\| \\
& \leqq \operatorname{card}\left(D_{m}\right) K^{n}\left(2^{-m} t\right)^{n} \varepsilon
\end{aligned}
$$

$$
D_{m}=\left\{r: \sum\left(r_{i}-1\right) t 2^{-m} \leqq t\right\}=\left\{r:|r| \leqq 2^{m}+n\right\}
$$

and $K$ is a constant satisfying

$$
\operatorname{tr}[\mathscr{J}(X, \varrho)] \leqq K \operatorname{tr}[\varrho]
$$

for all $\varrho \in V^{+}$. Therefore

$$
\begin{aligned}
\left\|\mathscr{E}_{m}^{t}(E, \varrho)-\mathscr{E}_{l}(E, \varrho)\right\| & \leqq(n !)^{-1}\left(2^{m}+n\right)^{n} K^{n} t^{n} 2^{-m n} \varepsilon \\
& =(n !)^{-1}\left(1+2^{-m} n\right)^{n} K^{n} t^{n} \varepsilon
\end{aligned}
$$

and

$$
\limsup _{l, m \rightarrow \infty}\left\{\left\|\mathscr{E}_{m}^{t}(E, \varrho)-\mathscr{E}_{l}^{t}(E, \varrho)\right\|\right\} \leqq(n !)^{-1} K^{n} t^{n} \varepsilon .
$$

As $\varepsilon>0$ is arbitrary we see that for each $\varrho \in V, \mathscr{E}_{m}^{t}(E, \varrho)$ forms a Cauchy sequence as $m \rightarrow \infty$, uniformly with respect to $E$. It is immediate that the limit function $\mathscr{E}^{t}(E, \varrho)$ is a bounded stochastic kernel. A similar estimate to Eq. (4.9) shows that for all $\varrho \in V^{+}$

$$
\operatorname{tr}\left[\mathscr{E}\left(A_{t}^{n}, \varrho\right)\right] \leqq(n !)^{-1} K^{n} t^{n} \operatorname{tr}[\varrho]
$$

which proves that $\mathscr{E}^{t}$ can be finitely defined for all Borel sets in $X_{t}$, satisfying

$$
\operatorname{tr}\left[\mathscr{E}\left(X_{t}, \varrho\right)\right] \leqq e^{K t} \operatorname{tr}[\varrho]
$$

for all $\varrho \in V^{+}$.

For all sets $E \cong A_{t}^{n}$ of the form

$$
E=\left\{\left(s_{n}, x_{n}, \ldots, s_{1}, x_{1}\right): t\left(r_{i}-1\right)<2^{m} s_{i} \leqq t r_{i} \quad \text { and } \quad x_{i} \in E_{i}\right\}
$$

it is easy to verify that

$$
\begin{aligned}
\mathscr{E}(E, \varrho)= & \int_{t\left(r_{\imath}-1\right)<2^{m_{S_{l}} \leqq t r_{\imath}}} S_{s_{n}} \mathscr{J}_{E_{n}} S_{s_{n-1}} \\
& \ldots \mathscr{J}_{E_{1}} S_{\left(t-s_{1}-\cdots-s_{n}\right)}(\varrho) d s_{1} \ldots d s_{n} .
\end{aligned}
$$

For fixed $E_{1}, \ldots, E_{n}$ the integrand is a jointly continuous function of $s_{1}, \ldots, s_{n}$ so it is easy to justify transforming the variables back to $t_{1}, \ldots, t_{n}$ to conclude that for all sets $F \leqq A_{t}^{n}$ of the form

$$
\begin{aligned}
F & =\left\{\left(x_{i}, t_{i}\right)_{i=1}^{n}: p_{i}<t_{i} \leqq q_{i} \text { and } x_{i} \in E_{i}\right\} \\
\mathscr{E} t(F, \varrho) & =\int_{p_{t}<t_{1} \leqq q_{i}} S_{t-t_{n}} \mathscr{J}_{E_{n}} S_{t_{n}-t_{n-1}} \ldots \mathscr{J}_{E_{1}} S_{t_{1}}(\varrho) d t_{1} \ldots d t_{n} .
\end{aligned}
$$


In this form it is easy to see that for two such sets $F_{1} \subseteq X_{s}$ and $F_{2} \subseteq X_{t}$

$$
\mathscr{E}^{\mathscr{S}+t}\left(\lambda\left(F_{1} \times F_{2}\right), \varrho\right)=\mathscr{E}^{S}\left(F_{1}, \mathscr{E}^{t}\left(F_{2}, \varrho\right)\right) .
$$

Since such sets form a semiring which generates the $\sigma$-field of all Borel subsets of $X_{t}$, it follows that $\mathscr{E}$ satisfies the generalised ChapmanKolmogorov equation (2.1). We can now construct the semigroup $T_{t}$.

It is clear that $\mathscr{E} t$ has $Z, \mathscr{J}$ as infinitesimal generators in the sense of Theorem 4.6. From Eqs. (4.10) and (4.13) we obtain

$$
T_{t}(\varrho)=S_{t}(\varrho)+t \mathscr{J}(X, \varrho)+o(t)
$$

as $t \rightarrow 0$. This shows that $\mathscr{E} t$ is strongly continuous in the sense of the definition of stochastic processes. Taking the trace of both sides of Eq. (4.14) and differentiating gives, by Lemma 4.5,

$$
\frac{d}{d t} \operatorname{tr}\left[T_{t}(\varrho)\right]=0
$$

for all $\varrho \in V$. It follows that $\operatorname{tr}\left[T_{t}(\varrho)\right]$ is constant, which concludes the proof that $\mathscr{E}^{\mathscr{t}}$ is a stochastic process.

Corollary 4.8. Both Theorems 4.6 and 4.7 are valid if $X$ is only a Borel subset of any complete separable metric space.

Proof. By [14] there exists a Borel isomorphism of $X$ onto $[0,1]$ or onto a subset of the integers. None of the conditions or conclusions of the theorems involve the topological structure of $X$.

\section{§ 5. Comments and Examples}

We have shown that the construction of certain quantum stochastic processes can be reduced to the choice of a suitable Hilbert space $\mathscr{H}$ and a pair $Z, \mathscr{J}$ of infinitesimal generators. In fact on physical grounds we can present what will usually be the most suitable candidate for $Z$. The process corresponds to a quantum system with a Hamiltonian $H_{0}$ say, which is perturbed by the introduction of the measuring instrument. If $R$ is the total interaction rate of $\mathscr{J}$ defined by

$$
\operatorname{tr}[R \varrho]=\operatorname{tr}[\mathscr{J}(X, \varrho)]
$$

then the operator $Z$ defined on $\mathscr{D}_{H_{0}}$ by $Z=i H_{0}-\frac{1}{2} R$ satisfies

$$
2 \operatorname{Re}\langle Z \xi, \xi\rangle=-\langle R \xi, \xi\rangle
$$

for all $\xi \in \mathscr{D}_{Z}$ and is the infinitesimal generator of a strongly continuous semigroup $B_{t}$ of contractions of $\mathscr{H}$ by [18].

If we make this choice for $Z$ then we are left only with the construction of $\mathscr{J}$. In [6] we undertook a detailed analysis of the structure of $\mathscr{J}$ for a 
position-measuring instrument. Taking $\mathscr{J}$ as in [6, Section 5] we obtain an approximate model of a position measuring apparatus such as a bubble chamber, where the events are bubbles in the chamber, thought of as points in space-time, and the sample points of the process are the chains of bubbles left by a quantum particle traversing the chamber. We do not claim that this is more than a crude model but is does demonstrate that such a system can be treated in a quantum mechanical manner.

Preparatory to considering more interesting examples, we introduce a fairly general class of bounded stochastic kernels. In the following theorem $X$ may be taken as the closure of an open bounded set in $\mathbb{R}^{2}$ with Lebesgue measure if the detector has a sensitised planar surface, or as a finite set with the counting measure, in the case where the detector consists of a collection of particle counters.

Theorem 5.1. Let $X$ be a separable compact Hausdorff space provided with a bounded Borel measure $\mu$ whose support is equal to $X$. Let $\mathscr{H}$ be a separable Hilbert space and $A_{x}$ a strongly continuous family of bounded operators on $\mathscr{H}$ defined for $x \in X$. Then the formula

$$
\operatorname{tr}[\mathscr{J}(E, \varrho) B]=\int_{E} \operatorname{tr}\left[A_{x} \varrho A_{x}^{*} B\right] \mu(d x)
$$

where $\varrho \in \mathscr{T}_{s}(\mathscr{H}), B \in \mathscr{L}_{s}(\mathscr{H})$ and $E \cong X$, defines a bounded stochastic kernel. Moreover the equation

$$
\mathscr{J}(E, \mathscr{J}(F, \varrho))=\mathscr{J}(F, \mathscr{J}(E, \varrho))
$$

holds for all $E, F \cong X$ and $\varrho \in \mathcal{T}_{s}(\mathscr{H})$ if and only if

$$
\left(A_{x} A_{y} \xi\right) \otimes\left(A_{x} A_{y} \xi\right)^{-}=\left(A_{y} A_{x} \xi\right) \otimes\left(A_{y} A_{x} \xi\right)
$$

for all $\xi \in \mathscr{H}$ and $x, y \in X$.

Proof. Since $X$ is compact and $\left\|A_{x} \xi\right\|$ is a continuous function on $X$ for all $\xi \in \mathscr{H}$, the uniform boundedness theorem implies that there exists a constant $K<\infty$ such that $\left\|A_{x}\right\| \leqq K$ for all $x \in X$. We observe that $x \rightarrow \operatorname{tr}\left[A_{x} \varrho A_{x}^{*} B\right]$ is always a continuous function on $X$. As in [6] we see that for each $\varrho \in V^{+}$and $E \subseteq X$ the right hand side of Eq. (5.1) defines a normal positive linear functional of $B$ and hence a positive trace-class operator $\mathscr{J}(E, \varrho)$ on $\mathscr{H}$ so that Eq. $(5.1)$ is satisfied. The proof that $\mathscr{J}$ is a bounded stochastic kernel proceeds exactly as in [6]. Since

$$
\operatorname{tr}\left[\mathscr{J}(E, \mathscr{J}(F, \varrho) B]=\int_{E} \int_{F} \operatorname{tr}\left[A_{x} A_{y} \varrho A_{y}^{*} A_{x}^{*} B\right] \mu(d x) \mu(d y)\right.
$$

it is clear that Eq. (5.3) implies Eq. (5.2). Conversely if $U_{n}, V_{n}$ are decreasing basic families of open neighborhoods of two points $x, y \in X$ then

$\operatorname{tr}\left[A_{x} A_{y} \varrho A_{y}^{*} A_{x}^{*} B\right]=\lim _{m, n \rightarrow \infty} \mu\left(U_{m}\right)^{-1} \mu\left(V_{n}\right)^{-1} \operatorname{tr}\left[\mathscr{J}\left(U_{m}, \mathscr{J}\left(V_{n}, \varrho\right)\right) B\right]$

which shows that Eq. (5.2) implies Eq. (5.3). 
We remark that if the $A_{x}$ are unbounded operators with a common dense domain then the same sort of result holds under reasonable conditions. The stochastic kernel will in this case not be bounded and will in fact only be well-defined for nice states. In the case where $X$ is finite, it is easy to formulate appropriate conditions.

An interesting case occurs when $X$ is the closure of an open relatively compact subset of a transitive $G$-space $Y$ which possesses an invariant measure, for example where $G=S O(3)$ (S) $\mathbb{R}^{3}$ and $Y=\mathbb{R}^{3}$. If $U$ is a unitary representation of $G$ on $\mathscr{H}$ then, generalising [6], we say that $\mathscr{J}$ is covariant with respect to $U$ if for all open sets $V \subseteq X$ such that $V_{g} \subseteq X$

$$
\mathscr{J}\left(V_{g}, \varrho\right)=U_{g}^{*} \mathscr{J}\left(V, U_{g} \varrho U_{g}^{*}\right) U_{g} .
$$

The corresponding condition on the operators $A_{x}$ is that for all $x \in X$ and $g \in G$ such that $x g \in X$

$$
A_{x g}=U_{g}^{*} A_{x} U_{g} .
$$

Now let us suppose that we have a quantum system consisting of a number of quantum particles and represented by a Hilbert space $\mathscr{H}$ with a Hamiltonian $H_{0}$. Let us introduce a detector which records the arrival of the particles on a sensitive surface $X$ and suppose that to each $x \in X$ we can associate a bounded operator $A_{x}$ which we shall call the annihilation operator corresponding to $x$ and such that for each $\varrho \in \mathscr{T}_{s}(\mathscr{H})^{+}$ and $x \in X, \operatorname{tr}\left[A_{x}^{*} A_{x} \varrho\right]$ gives the rate of arrival of particles at $x$ per unit area and time. We construct the bounded stochastic kernel $\mathscr{J}$ as described above, observe that the total interaction rate $R$ is

$$
R=\int_{X} A_{x}^{*} A_{x} \mu(d x)
$$

where $\mu$ is Lebesgue measure, define $Z=i H_{0}-\frac{1}{2} R$ and obtain the corresponding stochastic process from Theorem 4.7.

Now let us look more closely at the case where we have two identical non-directional, movable counters $C_{1}, C_{2}$ placed at points $x_{1}, x_{2}$ in some larger space $Y$ and suppose they are associated with the annihilation operators $A_{x_{1}}$ and $A_{x_{2}}$ respectively. Let us look at the event that in the initial state $\varrho$ there is exactly one count at $C_{1}$ in the time interval $(0, t]$ and exactly one count at $C_{2}$ in the interval $(t, 2 t]$ and no other counts in the interval $(0,2 t]$. The conditional output state at time $2 t$ for this event is

$$
t^{2} \mathscr{J}\left(x_{2}, \mathscr{J}\left(x_{1}, \varrho\right)\right)+o\left(t^{2}\right)=t^{2} A_{x_{2}} A_{x_{1}} \varrho A_{x_{1}}^{*} A_{x_{2}}^{*}+o\left(t^{2}\right) .
$$

Now for relativistic reasons we should be very surprised if the conditional output state for very small $t$ depended critically on which of the events occurred first, because for small enough $t$ the events will be causally unrelated. (Note that this does not imply that they are uncorrelated [3].) 
Causal arguments thus lead us to the conclusion that for all $x_{1} \neq x_{2} \in Y$ and all states $\varrho$ we should expect

$$
A_{x_{2}} A_{x_{1}} \varrho A_{x_{1}}^{*} A_{x_{1}}^{*}=A_{x_{1}} A_{x_{2}} \varrho A_{x_{2}}^{*} A_{x_{1}}^{*} .
$$

Moreover if $Y$ is a transitive $G$-space and $U$ is a unitary representation of $G$ on $\mathscr{H}$ we can restrict the annihilation operators $A_{x}$ obtained for all $x \in Y$ by moving the counters around to satisfy

for all $x \in Y, g \in G$.

$$
A_{x g}=U_{g}^{*} A_{x} U_{g}
$$

These equations have been derived subject to the assumption that $A_{x}$ are bounded, but we should expect them to hold in the general case also. I should like to thank I. T. Todorov for explaining to me the relation of the following result to axiomatic field theory.

Theorem 5.2. Let $G$ be a Lie group and $X$ a connected transitive $G$-space such that for all $x, y \in X$ there exists $g \in G$ satisfying $x g=y$ and $y g=x$. Let $U$ be a representation of $G$ on a separable Hilbert space $\mathscr{H}$ and suppose $\mathscr{D}$ is a dense $G$-invariant subspace of $\mathscr{H}$ provided with a topology stronger than the norm topology and such that $g, \xi \rightarrow U_{g} \xi$ is jointly continuous from $G \times \mathscr{D}$ to $\mathscr{D}$. Let $A_{x}$ be a family of continuous linear operators from $\mathscr{D}$ into $\mathscr{D}$ parametrised by $x \in X$ and such that for all $g \in G$ and $x \in X, A_{x g}=U_{g}^{*} A_{x} U_{g}$. Suppose that for all $x \neq y \in X$ and $\xi \in \mathscr{D}$

$$
\left(A_{x} A_{y} \xi\right) \otimes\left(A_{x} A_{y} \xi\right)^{--}=\left(A_{y} A_{x} \xi\right) \otimes\left(A_{y} A_{x} \xi\right)^{-}
$$

and that for all $x \neq y \in X$ there exists $\xi \in \mathscr{D}$ such that $A_{x} A_{y} \xi \neq 0$. Then either

or

$$
A_{x} A_{y}-A_{v} A_{x}=0 \quad \text { for all } \quad x, y \in X
$$

$$
A_{x} A_{y}+A_{y} A_{x}=0 \text { for all } x, y \in X .
$$

Remark. It is not possible by this approach to obtain any commutation relations between $A_{x}$ and $A_{y}^{*}$ because all the expressions involving these quantities occur in normal order automatically.

Proof. For all $x \neq y \in X$ and $\xi \in \mathscr{D}$ there exists a constant $\lambda$ with $|\lambda|=1$ such that

$$
A_{x} A_{y} \xi=\lambda A_{y} A_{x} \xi .
$$

Simple calculations show that this constant can be chosen independently of $\xi$ so that

for some function

$$
A_{x} A_{y}=\lambda(x, y) A_{y} A_{x}
$$

$$
\lambda: M \equiv\{(x, y) \in X \times X: x \neq y\} \rightarrow\{z \in \mathbb{C}:|z|=1\} .
$$


For each $x_{0} \neq y_{0}$ the value $\lambda\left(x_{0}, y_{0}\right)$ is unique by the non-degeneracy condition. If $\xi, \eta \in \mathscr{D}$ satisfy $\left\langle A_{x_{0}} A_{y_{0}} \xi, \eta\right\rangle \neq 0$ it follows from the equation

$$
\begin{aligned}
& \left\langle U_{g_{1}}^{*} A_{x_{0}} U_{g_{1}} U_{g_{2}}^{*} A_{y_{0}} U_{g_{2}} \xi, \eta\right\rangle= \\
& \quad=\lambda\left(x_{0} g_{1}, y_{0} g_{2}\right)\left\langle U_{g_{2}}^{*} A_{y_{0}} U_{g_{2}} U_{g_{1}}^{*} A_{x_{0}} U_{g_{1}} \xi, \eta\right\rangle
\end{aligned}
$$

that $\lambda$ is continuous in a neighbourhood of $\left(x_{0}, y_{0}\right)$. The transformation properties of $A_{x}$ imply that if $x \neq y$ then $\lambda(x, y)=\lambda(y, x)^{-1}$ and $\lambda(x g, y g)$ $=\lambda(x, y)$. It follows that $\lambda(x, y)=+1$. If $\operatorname{dim}(X) \geqq 2$ then as $M$ is obtained by removing a manifold of dimension $\operatorname{dim}(X)$ from a manifold of dimension $\operatorname{dim}(X)^{2}$, it must be connected and so $\lambda$ must be constantly either 1 or -1 . If $\operatorname{dim}(X)=1$ then either $X=\{z \in \mathbb{C}:|z|=1\}$ in which case $M$ is again connected, or $X=\mathbb{R}$. In the second case $M$ has two components but $x, y \rightarrow y, x$ interchanges them so $\lambda$ must again be constant.

We are now able to write down explicitly the stochastic process corresponding to a finite number of counters set up to measure the arrival of particles in a beam of fermions. We take $\mathscr{H}$ to be the antisymmetric Fock space built on $\mathscr{H}_{1}=\mathscr{L}^{2}\left(\mathbb{R}^{3}\right) \otimes \mathbb{C}^{2 n+1}$ so that

$$
\mathscr{H}=\mathbb{C} \oplus \mathscr{H}_{1} \oplus\left(\mathscr{H}_{1} \otimes \mathscr{H}_{1}\right)_{a} \oplus \cdots
$$

Let $x_{1}, \ldots, x_{n}$ be points in $\mathbb{R}^{3}$ representing the positions of the counters $C_{1}, \ldots, C_{r}$ respectively and let $f_{1}, \ldots, f_{n} \in \mathscr{H}_{1}$ be concentrated in small neighbourhoods of $x_{1}, \ldots, x_{n}$ respectively. Let $A: \mathscr{H}_{1} \rightarrow \mathscr{L}(\mathscr{H})$ be the mapping taking test functions in $\mathscr{H}_{1}$ to the bounded annihilation operators associated with the representation of the canonical anti-commutation relation on $\mathscr{H}$ (see [19]). We define the annihilation operator $A_{i}$ associated with the counter $C_{i}$ to be $A\left(f_{i}\right)$ so that $C_{i}$ is supposed to be sensitive to the field averaged over a small neighbourhood of $x_{i}$. We suppose the unperturbed Hamiltonian on $\mathscr{H}$ is $H_{0}$. Then $\mathscr{J}$ is defined by

and $Z$ is defined by

$$
\mathscr{J}(E, \varrho)=\sum_{i \in E} A_{i} \varrho A_{i}^{*}
$$

$$
Z=i H_{0}-\sum_{i=1}^{n} A_{i}^{*} A_{i}
$$

The stochastic process $\mathscr{E} t$ may now be constructed by Theorem 4.7 .

The corresponding theory for a beam of bosons runs into difficulties because the annihilation operators are unbounded and so the corresponding stochastic processes will have unbounded total interaction rates. Although these processes do not generally fall within the scope of this paper, we can by good fortune treat one very special case, where the particles have no interaction with each other. 
Take $\mathscr{H}$ to be the symmetric Fock space built on $\mathscr{H}_{1}=\mathscr{L}^{2}\left(\mathbb{R}^{3}\right) \otimes \mathbb{C}^{2 n}$ so that

$$
\mathscr{H}=\mathbb{C} \oplus \mathscr{H}_{1} \oplus\left(\mathscr{H}_{1} \otimes \mathscr{H}_{1}\right)_{s} \oplus \cdots .
$$

Let counters $C_{1}, \ldots, C_{n}$ be located at $x_{1}, \ldots, x_{n}$; let $f_{1}, \ldots, f_{n}$ be $C^{\infty}$ test functions in $\mathscr{H}_{1}$ with supports in small neighbourhoods of $x_{1}, \ldots, x_{n}$; let $A$ be the annihilation operator for the representation of the canonical commutation relations on Fock space [20]; and define the annihilation operators $A_{1}, \ldots, A_{n}$ for the counters $C_{1}, \ldots, C_{n}$ to be $A\left(f_{1}\right), \ldots, A\left(f_{n}\right)$ respectively. Let $H_{1}$ be the appropriate Hamiltonian for the motion of a single particle in $\mathscr{H}_{1}$ and let $H_{0}$ be the corresponding Hamiltonian on $\mathscr{H}$ which leaves each of the subspaces $\left(\mathscr{H}_{1} \otimes \cdots \otimes \mathscr{H}_{1}\right)_{s}$ invariant.

If $\mathscr{H}_{n}$ is the subspace of $\mathscr{H}$ corresponding to $\leqq n$ particles, then $\mathscr{H}_{n} \subseteq \mathscr{H}_{n+1}$ and $\mathscr{H}_{n}$ is invariant under $H_{0}$. Moreover $A_{i}: \mathscr{H}_{n} \rightarrow \mathscr{H}_{n-1}$ and are bounded on $\mathscr{H}_{n}$. Therefore we can use the same construction as in the fermion case to construct a stochastic process $\mathscr{E}_{n}^{t}$ with a bounded interaction rate on $\mathscr{H}_{n}$. The $\mathscr{E}_{n}^{t}$ are compatible in the sense that if $\varrho \in \mathscr{T}_{s}\left(\mathscr{H}_{n}\right)$ $\subseteq \mathscr{T}_{s}\left(\mathscr{H}_{n+1}\right)$ and $E \subseteq X_{t}$ then

$$
\mathscr{E}_{n}^{t}(E, \varrho)=\mathscr{E}_{n+1}^{t}(E, \varrho) \text {. }
$$

The construction is completed by proving that $\mathscr{E}_{n}^{t}$ have a common extension to $\mathscr{H}$.

Theorem 5.3. Let $X$ be a separable locally compact Hausdorff space and $\left\{\mathscr{H}_{n}\right\}_{n=1}^{\infty}$ an increasing sequence of closed subspaces with dense union in a separable Hilbert space $\mathscr{H}_{0}$. If $V_{n}=\mathscr{T}_{s}\left(\mathscr{H}_{n}\right)$ and $\mathscr{E}_{n}, n=1,2, \ldots$ is a sequence of compatible quantum stochastic processes on $X, V_{n}$ respectively then there exists a unique stochastic process $\mathscr{E}_{0}^{\text {t }}$ on $X, V_{0}$ which extends each of the $\mathscr{E}_{n}$.

Proof. The injection $i: \mathscr{H}_{n} \rightarrow \mathscr{H}_{n+1}$ induces an isometric injection $i: V_{n} \rightarrow V_{n+1}$ and the union $W=\bigcup_{n=1}^{\infty} V_{n}$ is a dense subspace of $V_{0}$. Moreover $W$ is positively generated and $W^{+}=\bigcup_{n=1}^{\infty} V_{n}^{+}$is dense in $V_{0}^{+}$. If $\mathscr{J}^{t}$ is defined as the common extension of $\mathscr{E}_{n}^{t}$ to $X, W$ then for each $t \geqq 0$ and $E \subseteq X_{t}, \varrho \rightarrow \mathscr{J}^{t}(E, \varrho)$ is a positive linear map of $W$ into $W$ of norm one and so has a unique extension to a positive linear map of norm one of $V_{0}$ into $V_{0}$. That is there exists a function $\mathscr{E}_{0}^{t}$ such that for each $t \geqq 0$, $E \subseteq X_{t}$ and $\varrho \in V_{0}^{+}, \mathscr{E}_{0}(E, \varrho) \in V_{0}^{+}$, and for each $t \geqq 0, E \subseteq X_{t}$ and $\varrho \in V_{n}$

$$
\mathscr{E}_{0}^{t}(E, \varrho)=\mathscr{E}_{n}^{t}(E, \varrho) \text {. }
$$

It is easy to verify that $\mathscr{E}_{0}^{t}$ satisfies all the axioms for a stochastic process, by making use of the fact that the operators $\varrho \rightarrow \mathscr{E}_{n}(E, \varrho)$ are uniformly bounded independently of $n, t$, and $E$. 


\title{
References
}

1. Glauber, R. J.: Photon statistics; Fundamental problems in statistical mechanics II, ed. by E. G. D. Cohen. Amsterdam: North-Holland Publishing Company 1968.

2. Klauder, J. R., and E. C. G. Sudarshan: Fundamentals of quantum optics. New York: W. A. Benjamin Inc. 1968.

3. Glauber, R. J.: Quantum theory of optical coherence. Phys. Rev. 130, 2529-2539 (1963).

4. Davies, E. B., and J. T. Lewis: An operational approach to quantum probability. To appear.

5. Davies, E. B.: Systems of imprimitivity. To appear.

6. - On the repeated measurement of continuous observables in quantum mechanics. To appear.

7. Feller, W.: An introduction to probability theory and its applications, Vol. 2. New York: Wiley 1966.

8. Mackey, G. W.: Unitary representations of group extensions. Acta Math. 99, 265-311 (1958).

9. Namioka, I.: Partially ordered linear topological spaces. Mem. Am. Math. Soc. 24 (1957).

10. Stormer, E.: Positive linear maps of operator algebras. Acta Math. 110, 233-278 (1963).

11. Hille, E., and R. S. Phillips: Functional analysis and semigroups. Coll. Publ. Am. Math. Soc. (1957).

12. Yoshida, K.: Functional analysis. Berlin-Heidelberg-New York: Springer 1965.

13. Dixmier, J.: Dual et quasi-dual d'une algèbre de Banach involutive. Trans. Am. Math. Soc. 104, 278-283, (1962).

14. Mackey, G. W.: Borel structure in groups and their duals. Trans. Am. Math. Soc. 85, 134-165 (1957).

15. Iwasawa, K.: On some types of topological groups. Ann. Math. 50, 507-558 (1949).

16. Bargmann, V.: On unitary ray representations of continuous groups. Ann. Math. 59, 1 - 46 (1954).

17. Halmos, P. R.: Measure theory. New York: D. van Nostrand 1950.

18. Lumer, G., and R. S. Phillips: Dissipative operators in a Banach space. Pacific J. Math. 11, 679-698 (1961).

19. Segal, I. E.: Tensor algebras over Hilbert spaces II. Ann. Math. 63, 160-175 (1956).

20. - Tensor algebras over Hilbert spaces I. Trans. Am. Math. Soc. 81, 106-134 (1956).

\author{
E. B. Davies \\ Institute for Advanced Study \\ Princeton, New Jersey, U.S.A. \\ Permanent Address: \\ Mathematical Institute \\ 24-29 St. Giles \\ Oxford, England
}

